\title{
NanoCarb hyperspectral sensor: on performance optimization and analysis for greenhouse gas monitoring from a constellation of small satellites
}

\author{
Silvère Gousset ${ }^{1}$ (D) - Laurence Croizé ${ }^{2} \cdot$ Etienne Le Coarer ${ }^{1} \cdot$ Yann Ferrec $^{2} \cdot$ Juana Rodrigo-Rodrigo $^{1} \cdot$ Laure Brooker $^{3}$. \\ for the SCARBO consortium
}

Received: 18 February 2019 / Revised: 1 August 2019 / Accepted: 26 August 2019 / Published online: 9 September 2019

(c) The Author(s) 2019

\begin{abstract}
NanoCarb is an innovative Fourier-transform imaging spectrometer dedicated to the measurement of $\mathrm{CO}_{2}$ and $\mathrm{CH}_{4}$. Both its unusual optical principle and sampling strategy allow to reach a compact design, ideal for small satellite constellation as investigated by the European project SCARBO. The NanoCarb performance assessment as well as a proof of concept is required in this framework. We have developed a design strategy to optimize the performances. We demonstrate the potential of the concept through an estimation of the sensitivity, compliant with the space mission target. We also present a preliminary mitigation of the bias induced by water on $\mathrm{CO}_{2}$ and $\mathrm{CH}_{4}$ retrieval, illustrating the efficiency and the flexibility of the NanoCarb partial interferogram sampling technique. The presented design reaches a sub-ppm random error for $\mathrm{CO}_{2}$ and sub-10 ppb random error for $\mathrm{CH}_{4}$, considering $128 \mathrm{~km}$ swath and 2 by $2 \mathrm{~km}^{2}$ ground resolution. Design optimization and more systematic performances are discussed.
\end{abstract}

Keywords Hyperspectral sensor · Anthropogenic GHG emissions $\cdot$ Fourier-transform spectroscopy $\cdot$ Near-IR passive remote sensing

\section{Introduction}

One of the issues of studying climate change is to reduce uncertainties in estimating emissions of the main greenhouse gasses (GHG) - $\mathrm{CO}_{2}$ and $\mathrm{CH}_{4}[1]$ - leading to a better distinction between anthropogenic and natural sources. The first challenge for dedicated space missions is to monitor the atmospheric total column and/or vertical profiles with both a drastically improved statistical error and smaller systematic

Silvère Gousset

silvere.gousset@univ-grenoble-alpes.fr

for the SCARBO consortium

http://scarbo-h2020.eu/

1 Institut de Planétologie et d'Astrophysique de Grenoble, Université Grenoble-Alpes, 38058 Grenoble, France

2 ONERA/DOTA, chemin de la Hunière, 80100, 91123 Palaiseau, France

3 Airbus Defence and Space, 31, rue des Cosmonautes, 31402 Toulouse, France bias. The second one is to provide an improved revisit frequency and spatial coverage of the monitoring.

LIDAR active technology (MERLIN European project [2] or ASCENDS project in the US [3]) achieves sensitive measurements independently to solar illumination conditions, in like manner dedicated spaceborne Thermal InfraRed (TIR) passive spectrometers (IASI [4]). Nevertheless, LIDAR are inherently limited to unidirectional sounding, while TIR spectrometers are less sensitive to the lowest atmospheric layers where anthropogenic activities are significant. By the opposite, Short Wave Infrared (SWIR or near IR) spectrometry only allows daytime monitoring, but with improved sensitivity to the lowest parts of the atmosphere. GOSAT [5] and GOSAT- $2,{ }^{1}$ OCO-2 (see footnote 1) or TANSAT (see footnote 1), are current space missions dedicated to $\mathrm{CO}_{2}$ and/or $\mathrm{CH}_{4}$ monitoring in this domain. Geo-CARB (see

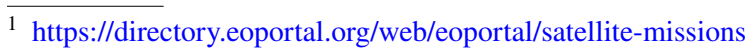


footnote 1) in the US, or MicroCarb [6], CO2M (CarbonSat) [7], and TROPOMI (see footnote 1) in Europe are the future planned missions or spaceborne instruments.

Nevertheless, not all these missions can achieve sufficient spatial and temporal coverage, with a typical revisit frequency of 15 days for the majority of them, from a sunsynchronous orbit. In addition, individual costs of these missions are a real hurdle to put into orbit a constellation.

A way forward to increase the revisit frequency and spatial coverage is to complete these current reference missions with a constellation of low cost, small satellites, and calling for breakthroughs in compact hyperspectral sensing. The challenge is to adapt kilogram-class payload for typical $50 \mathrm{~kg}$-class platforms, maintaining their performances compatible with current reference missions and the main science issues: kilometric ground resolution, sensitivity bellow 1 part per million (ppm) over the averaged dry column of $\mathrm{CO}_{2}$, and bellow 10 parts per billion (ppb) for $\mathrm{CH}_{4}$. Moreover, it is important to reach a $100 \mathrm{~km}$ swath to increase the spatial coverage and to contextualize some hotspots according to the background (e.g., plume).

In this framework, the Horizon 2020 project Space CARBon Observatory (SCARBO) [8] aims at assessing the feasibility of a low-cost constellation of small satellites onboarding dedicated GHG sensor. The SPEX [9] dedicated aerosols sensor is also provided in the platform. The project relies on small satellites to monitor $\mathrm{CO}_{2}$ and $\mathrm{CH}_{4}$ emissions, complementing the low-revisit high-performance satellites.

Several orbital configurations are investigated in this scenario. One of the simplest consists to put 40 small satellites into the same sun-synchronous orbit at $600 \mathrm{~km}$ height. With a $128 \mathrm{~km}$ swath and a small recovering between consecutive footprints at ground for cross-calibration purposes, it is possible to ensure a daily revisit over emerged lands at mid-latitudes.

The core miniature GHG sensor of this constellation is the NanoCarb concept, a static Fourier-transform imaging spectrometer, first introduced in sections III.B and III.C of [10]. In this concept, both the use of a low finesse Fabry-Perot array and a partial interferometric sampling strategy allow to achieve a large swath at high spectral resolution as well as to obtain an optimal use of the available pixels for a high sensitivity in a snapshot acquisition mode.

However, this uncommon instrumental concept enforces a shift of paradigm in atmospheric sounding domain. Indeed, the acquired data are quite different from classic dispersive or Fourier-based spaceborne systems: the information content of the data is very low (as defined in [11]), compared with a conventional radiance spectrum. Consequently, a careful upstream design of the instrument is required. The main issue is to maximize the sensitivity to the average column of $\mathrm{CO}_{2}$ and $\mathrm{CH}_{4}$, while minimizing biases due to other geophysical parameters.
In this paper, we introduce a forward approach to design NanoCarb, and then we analyze its performances for $\mathrm{CO}_{2}$ and $\mathrm{CH}_{4}$ atmospheric total column measurement. We base this approach on an analytical model of the NanoCarb radiometric sensitivity coupled with considerations over information present in the Fourier domain. We present a preliminary design of the two main components of the instrument which are the narrowband filter and the interferometer, and we investigate some promising features of the concept.

In Sect. 2, we present the NanoCarb principle and sampling strategy. Then in Sect. 3, we describe our radiometric model (direct or forward model), which is used in Sect. 4 to select the optimal spectral band, and in Sect. 5 to select the optical path differences (OPD). We derive and discuss some considerations about performance capabilities of the concept.

\section{Nanocarb concept principle}

In this section, we describe the principle of the NanoCarb spectrometer: optical principle and sampling strategy. A description of the expected data products is also presented, to introduce some potential calibration or data processing issues, even though the purpose of this paper is only focused on theoretical description and preliminary design.

\subsection{Optical principle}

NanoCarb is a static Fourier-transform imaging spectrometer. We can distinguish two optical sub-systems: the front optics and the interferometric core, as it can be seen in Fig. 1.

The front optics consist of an afocal system-(2) and (4) - with a field stop (3), allowing to adapt the Fieldof-View (FoV) independently from the interferometric core (5-7). A baffle (1) stays in front of the afocal system. An interference filter (5) selects the spectral band. Four independent spectral bands have been currently identified for SCARBO as reported in Table 1. In the scope of this paper, we consider only the $\mathrm{B} 2$ and $\mathrm{B} 3$ bands to optimize $\mathrm{CO}_{2}$ and $\mathrm{CH}_{4}$ sensitivity, assuming a clear sky and a perfect measurement of the atmospheric pressure, thanks to band B1.

The interferometric core is formed by the association of an interferometer array (6) with a microlens array ( $\mu$ lens-7). Each interferometer has a specific thickness. The focal plane array (FPA-8) is placed in the focal plane of the $\mu$ lens array, to obtain a set of thumbnails, each one being associated to one thickness of the interferometric plate. In this configuration, the image formed in each thumbnail is a replication of the same FoV, modulated by the associated interferometer.

Each interferometer is a low-finesse Fabry-Perot (FP), which modulates the spectrum of the incoming light in accordance with the interferometer optical path difference (OPD), 
Fig. 1 Optical principle of NanoCarb

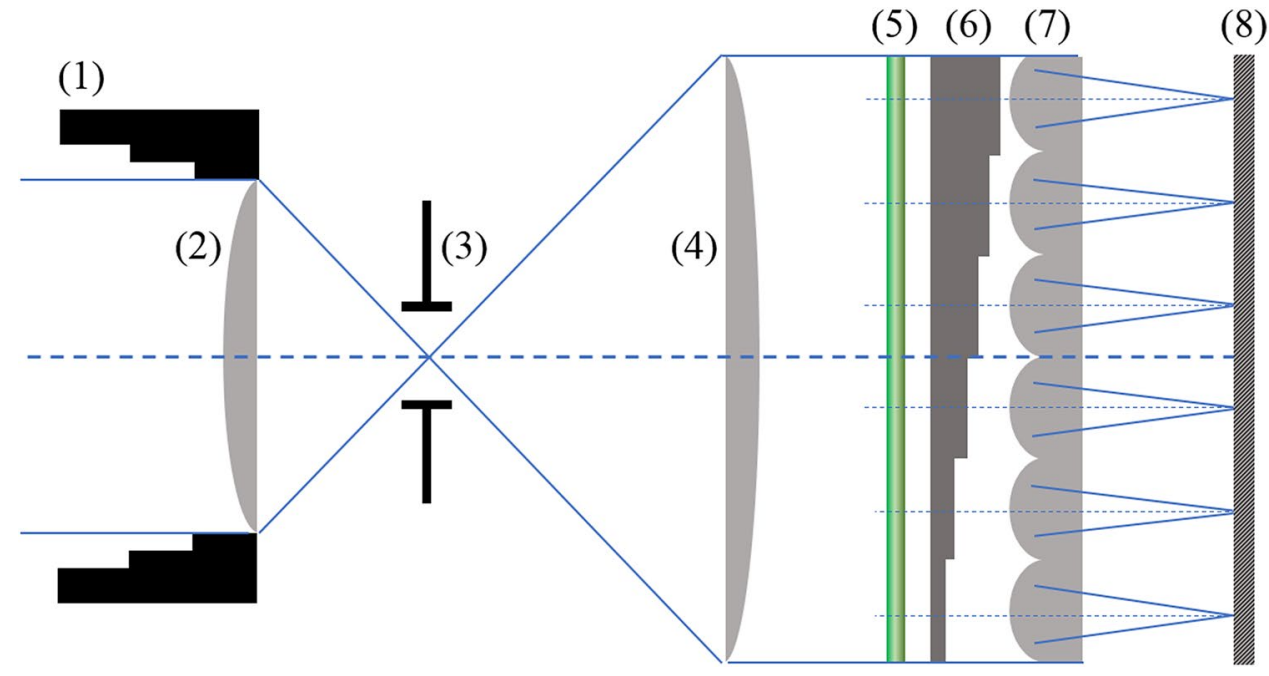

Table 1 Current NanoCarb bands

\begin{tabular}{lll}
\hline Band & Region & Measurement \\
\hline $\mathrm{B} 1-\mathrm{O}_{2}$ & $760 \mathrm{~nm}$ & $\begin{array}{c}\text { Surface } \\
\text { pressure, } \\
\end{array}$ \\
& & aerosols \\
$\mathrm{B} 2-\mathrm{CO}_{2}$ & $1.6 \mu \mathrm{m}$ & $\mathrm{CO}_{2}$ \\
$\mathrm{~B} 3-\mathrm{CH}_{4}$ & $1.66 \mu \mathrm{m}$ & $\mathrm{CH}_{4}$ \\
B4-strong $\mathrm{CO}_{2}$ & $2.06 \mu \mathrm{m}$ & Aerosols \\
\hline
\end{tabular}

which depends mainly on the Fabry-Perot thickness and slightly on the angle of incidence (this latter dependency creating the rings which modulate the image on each thumbnail, see Fig. 4). The currently investigated device is based on silicon, offering a natural reflection at the air-Si interface about 55\%, which leads to two-wave-dominated interferences. This configuration is optimal to manage at the same time the compactness of the device and large $\mathrm{FoV}$, as well as sensitivity as explained in [12] with more details. The device can be replicated, each one filling a part of the same FPA or using a dedicated FPA, to monitor synchronously several spectral bands.

Because interferential filter lies in the pupil plane in front of $\mu \mathrm{FP}$ array, a spectral band shift occurs in the field of view of each thumbnail. We integrate this shift in the NanoCarb numerical model and consider it in the design of the instrument.

This concept is compact and fully static. In the following, we will explain the partial interferometric sampling strategy of the NanoCarb concept, enabling snapshot acquisitions at high spectral resolution.

\subsection{Partial sampling of the interferogram}

The strategy we adopt to reach a high spectral resolution while keeping a large swath in snapshot mode is to target only the useful information in the Fourier space. Thus, the NanoCarb spectrometer acquires only partial interferograms.

The acquisition of partial interferograms was already developed in the 1970s by Kyle for temperature measurement in $\mathrm{CO}_{2}$ lines [13], then by Fortunato who applied this method to the measurement of $\mathrm{SO}_{2}$ concentration [14]. More recently, some studies can be found for the SIFTI instrument [15], and for IASI data processing [16], showing both a better geophysical bias mitigation, such as surface temperature, and an improvement of the signal-to-noise ratio (SNR).

In the case of NanoCarb, the spectral band is purposely selected to optimize the sensitivity over targeted regions of the interferogram, leading to an optical filtering of the useless information (interferants) both in the spectral and in the Fourier domain. An interferential filter as introduced in the previous sub-section is used to select the spectral band (spectral filtering), in front of the $\mu$-interferometer array (interferometric filtering). This principle is well suited for $\mathrm{CO}_{2}$ and $\mathrm{CH}_{4}$, thanks to a periodic spectral pattern over specific bands, for example on the $1.6 \mu \mathrm{m}$ band, or on the $1.66 \mu \mathrm{m}$ band for $\mathrm{CH}_{4}$ as visible in Fig. 2. In these conditions, the signature of these two species in the Fourier domain is well concentrated as visible in Fig. 3.

Hence, the aim of the partial interferogram sampling is:

- To obtain a measure of the $X$-specie concentration, as independently of the interferants as possible (for example, water).

- To obtain a measure of the interferants where appropriate, as independently as possible. The main atmospheric interferants are the aerosols and the water vapor. Temperature profile, pressure at ground, and albedo are also crucial in the retrieval process, and consequently require dedicated spectral or interferometric channels.

Figure 3 shows some partial derivative interferograms for a variation of concentration of $\mathrm{CO}_{2}, \mathrm{CH}_{4}$, and water, for the 


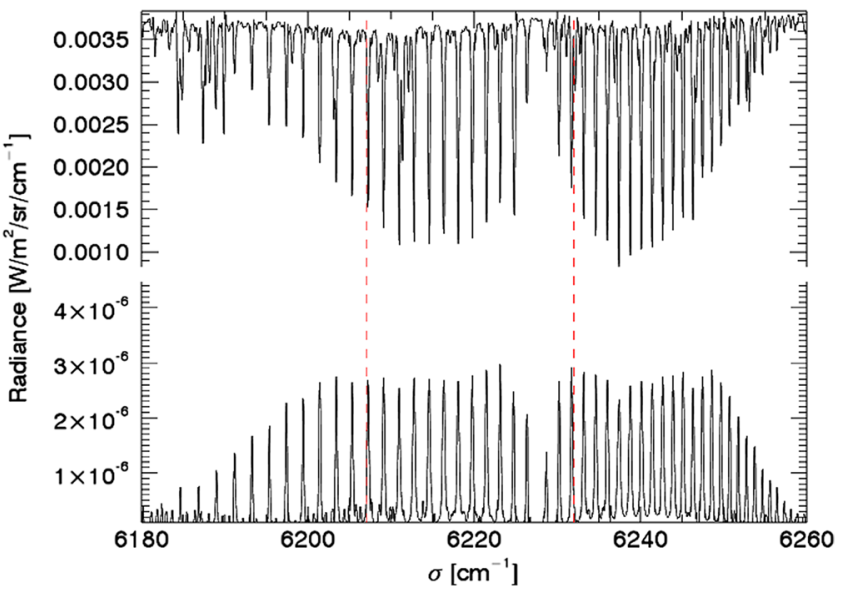

Fig. 2 Simulated spectral radiance (top curve) and partial derivative radiance (bottom curve). Left: on $1.6 \mu \mathrm{m} \mathrm{CO}_{2}$ band, with a variation of $1 \mathrm{ppm}$ of $\mathrm{CO}_{2}$ over the total column for the derivative radiance. Right: on $1.66 \mu \mathrm{m} \mathrm{CH}_{4}$ band, with a variation of $10 \mathrm{ppb}$ of $\mathrm{CH}_{4}$. The

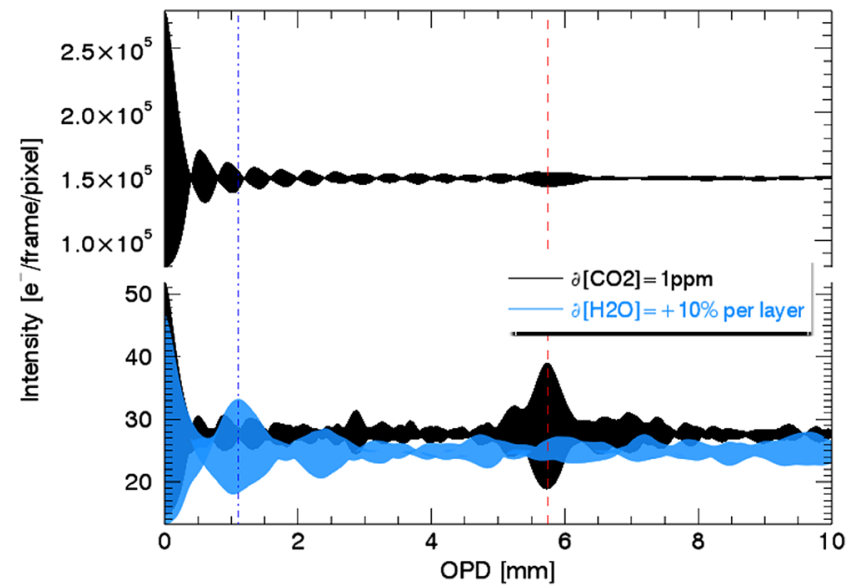

Fig. 3 Interferogram (top curve) and partial derivative interferograms (bottom curve). Left: for the $\mathrm{CO}_{2}$ band, with a variation of $1 \mathrm{ppm}$ of $\mathrm{CO}_{2}$ for the black-plotted derivative. Right: for the $\mathrm{CH}_{4}$ band, with a variation of $10 \mathrm{ppb}$ of $\mathrm{CH}_{4}$ for the black-plotted derivative; in blue,

spectral band chosen as a example. The red dashed lines highlight the regions where the sensitivity of $\mathrm{CO}_{2}$ or $\mathrm{CH}_{4}$ is optimal, but biased by water, while the blue ones spot regions where the measurement of water is optimal with respect to $\mathrm{CO}_{2}$ or $\mathrm{CH}_{4}$.

Figure 4 shows how with a controlled distribution of thickness over the FP array we can sample the interferogram at targeted OPD, potentially through disjoint interferometric regions. For a given region, the thicknesses are chosen to achieve a continuous $\lambda / 4$-sampling. First, this allows to obtain a good sampling of the interferogram on a single acquisition, and thus enables snapshot acquisition mode; second it is more convenient for fringe contrast estimation based on generalized ABCD techniques [17].

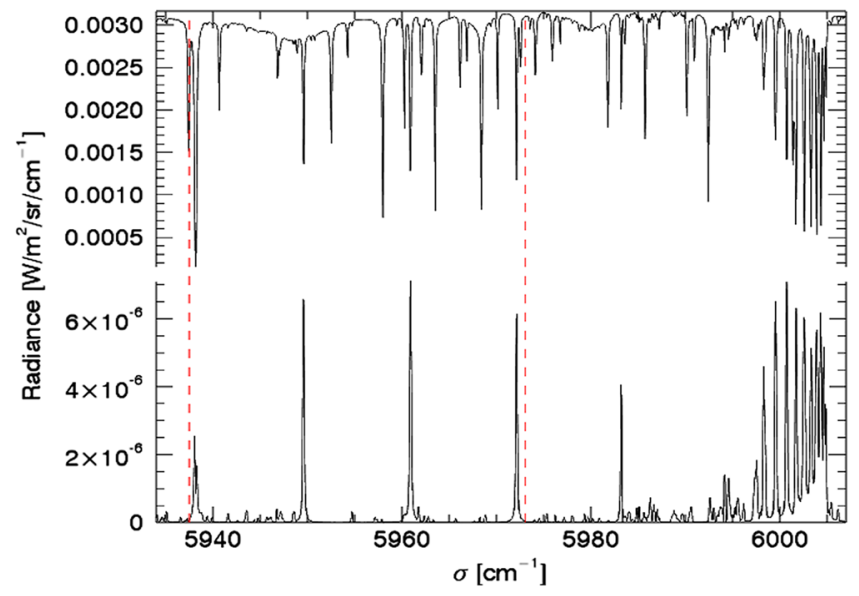

spectral regions between the red dashed lines allow to optimize the sensitivity of NanoCarb and are targeted by the relevant interferometric filter

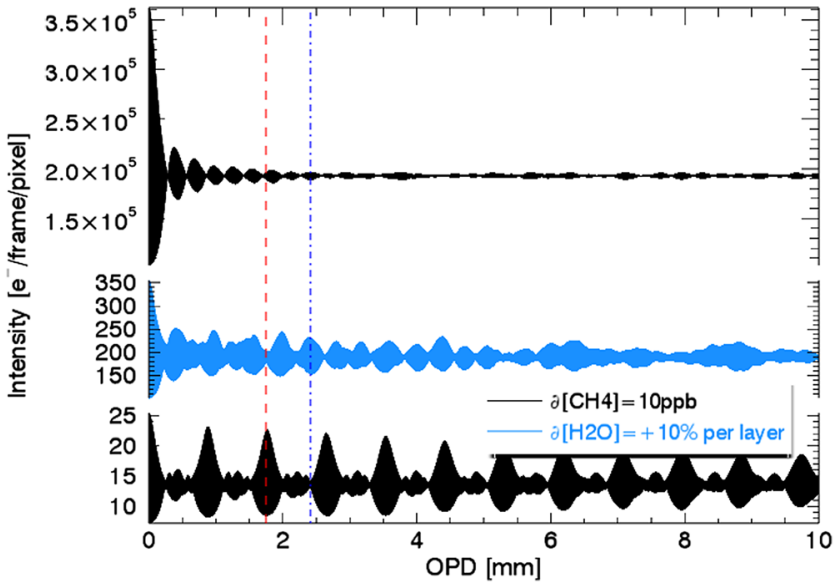

the partial derivative for a variation of $10 \%$ of the total column of water for each band. The red dashed lines show the OPD where the sensitivity is optimal. The blue dotted dashed lines show a maximum of sensitivity to water. Albedo $=0.2$, solar angle $=20^{\circ}, \mathrm{iFoV}=2 \mathrm{~km}$

A particular attention must be paid to the choice of spectral bands and sampled OPDs when designing NanoCarb. These points will be detailed, respectively, in Sects. 4 and 5 .

\subsection{NanoCarb data products}

The LO data product of NanoCarb is the snapshot focal plane intensity acquired by the detector. For demonstration purpose, a noiseless simulation can be seen in Fig. 4-left, in the unrealistic case of a spatially and spectrally uniform scene.

The colored points spot an individual field of view ( $\mathrm{iFoV}$ ) imaged in all the thumbnails. The extraction of the intensity 


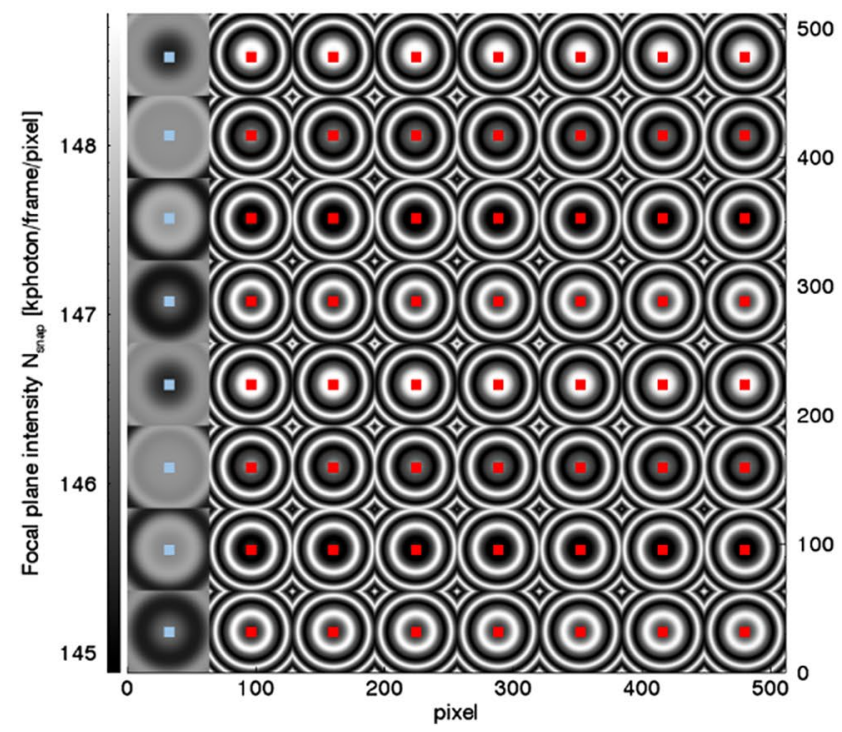

Fig. 4 Left: simulated focal plane intensity of NanoCarb in the $1.6 \mu \mathrm{m} \mathrm{CO}_{2}$ band, equivalent to L0 data product. Right: extracted snapshot interferogram for the spotted $\mathrm{iFoV}$, equivalent to L1a data product. In this configuration, we target two disjoint interferometric

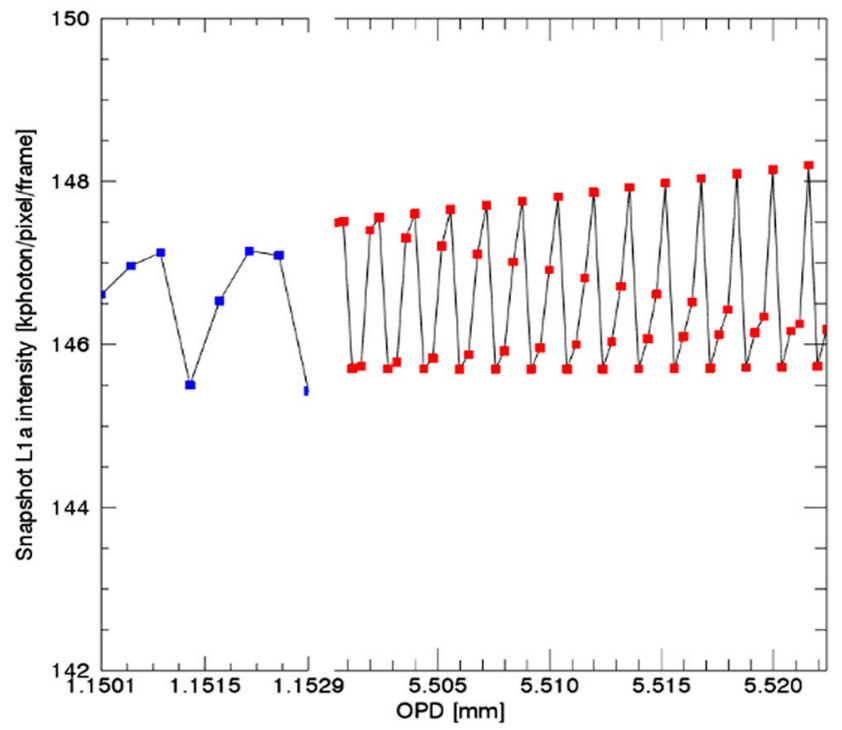

regions. The left thumbnail column is dedicated to the sampling of particularly water-sensitive fringes, while the rest of the thumbnails are dedicated to $\mathrm{CO}_{2}$
Fig. 5 iFoV shifting across the thumbnail as a function of time. The right image illustrates how the intensity is modulated over each exposure with the FabryPerot rings crossing. In that way, we can measure several interferometric states for this $\mathrm{iFoV}$

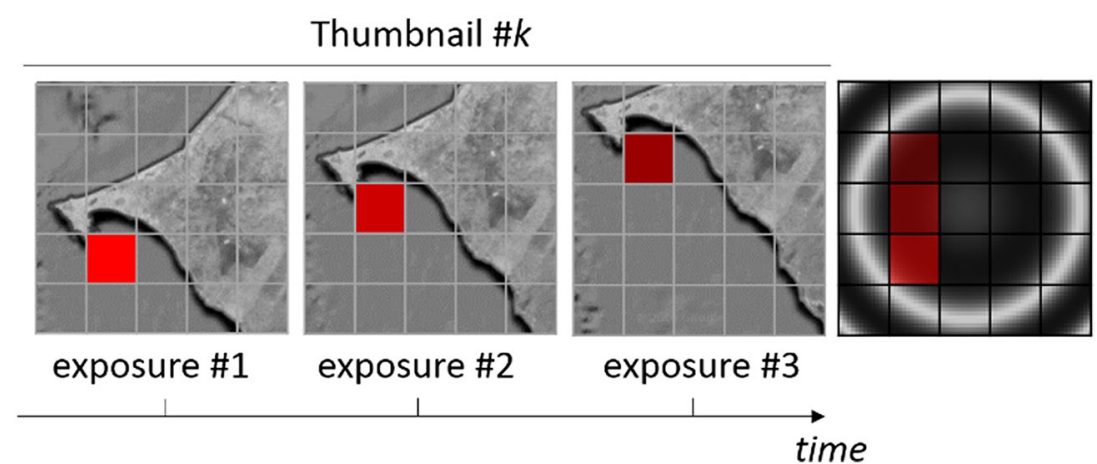

on a single exposure for this $\mathrm{iFoV}$ allows to retrieve the associated partial interferogram (Fig. 4-right), assuming a lab calibration of the corresponding OPD (see [18] and [19]). This snapshot interferogram is the L1a data product of NanoCarb. For a given iFoV at this level, $n_{\mathrm{FP}}$, the number of thumbnails - or FP_per spectral band, is the number of interferometric channels.

The L1b data product is the final vector of measures to be inversed. It is the concatenation of all the consecutive snapshot exposures during the scan of the scene as illustrated in Fig. 5. Between each exposure, the interferometric state changes (Fabry-Perot rings), as well as the spectral response of the pixel. This latter is due to the spectral shift of the interferential filter in the field. For this reason, a representation of a $\mathrm{L} 1 \mathrm{~b}$ interferogram makes no sense on the OPD scale used in Fig. 4. This co-registration of snapshot acquisitions is crucial to reach a sufficient SNR as we will see later in this paper. For a given $\mathrm{iFoV}$ at this level, $n_{\mathrm{FP}} \times N_{\mathrm{s}}$ is the maximum number of interferometric channels per spectral band, with $N_{\mathrm{s}}$ the number of pixels by side of thumbnail (assuming that the satellite has moved one iFoV forward between two frames).

The $L 2$ data product is the concentration value for a single iFoV, retrieved from this L1b concatenated partial interferogram. In the case of a multi-band design, the measurement vector to be inversed is the concatenation of all the L1b concatenated partial interferograms from each one of these bands.

\section{Analytical radiometric model of performances}

We propose here a solution to estimate the sensitivity of NanoCarb as a function of the instrumental parameters. Model of intensity over the FPA at each level of data 
is introduced as well as derived analytical performance expression. In this model, the scene is assumed to be homogeneous at the scale of the spatial resolution.

\subsection{NanoCarb instrumental model}

We use a simple analytical model of the NanoCarb intensity over the focal plane, derived from the approximate expression of the transmission of a Fabry-Perot assuming Fresnel reflections at the interfaces. This normalized transmission in intensity for a monochromatic radiation is:

$T_{\mathrm{FP}} \approx \frac{1}{1+M \sin ^{2}\left(\frac{\varphi}{2}\right)}$,

$M=4 R /(1-R)^{2}$ is the finesse term depending on the reflectivity at the FP interfaces $R$ and $\varphi$ the phase shift between two consecutive transmitted rays:

$\varphi=2 \pi \sigma \delta$,

where $\sigma$ is the wavenumber and $\delta$ is the OPD.

A weighted integration of Eq. (1) over the considered spectral band $\Delta \sigma$ allows to derive the number of electrons per pixel on a single frame on the detector $N_{\text {snap: }}$ :

$N_{\text {snap }}=\eta \int_{\Delta \sigma} \frac{(1 / \mathrm{hc} \sigma) T_{\sigma} L_{\sigma}}{1+M_{\sigma} \sin ^{2}\left(\frac{\varphi(\sigma)}{2}\right)} d \sigma,[\mathrm{e} /$ frame/pixel $]$,

where $L_{\sigma}$ is the spectral terrestrial radiance (see next subsection), $T_{\sigma}$ the normalized spectral transmission of the instrument including, for instance, detector quantum efficiency and interferential filter spectral shape, and $\eta$ is the $\sigma$-independent radiometric term, gathering exposure time $t_{\text {exp }}$, single pixel optical etendue $\Omega S$, and panchromatic optical transmission of the instrument $T_{\text {opt }}$ :

$\eta=\Omega S t_{\mathrm{exp}} T_{\mathrm{opt}},\left[\operatorname{strd} . m^{2} . s\right]$.

Equations (1-4) allow to simulate 1D-interferograms with NanoCarb radiometric properties on an arbitrary scale of OPD as presented in Fig. 3.

Such a kind of interferogram is very convenient to explore information content and sensitivity in the Fourier domain, by deriving fringe visibility $V$. The visibility trend with the OPD, $V(\delta)$, paints the interferometric shape or envelope of the interferogram. This latter is estimated as follows:

$V(\delta)=F(\delta) / \bar{N}$,

where $F(\delta)$ is the coherent flux at $\delta$ in electron per frame and per pixel and $\bar{N}$ is the mean level of the interferogram in the same unit. Band optimization and interferometric region targeting described in the following sections are based on this envelope estimation.

\subsubsection{On NanoCarb data product models}

We can easily simulate 2D-images of the NanoCarb L0 data product (e.g. Fig. 4) from Eq. (3) by implementing NanoCarb geometry, including FP thickness distribution and angular dependency in the field of view of each thumbnail. The main feature is an OPD variation in the FoV responsible for FP ring patterns, explicitly stated in the following expression:

$\delta(\sigma, i, \theta, T)=2 n(\sigma, T) \varepsilon_{\mathrm{i}} \cos \theta_{\mathrm{r}}$,

where $T$ is the temperature, $\varepsilon_{\mathrm{i}}$ the mechanical thickness of the ith FP, $n$ the optical refractive index of the FP material, and $\theta$ and $\theta_{\mathrm{r}}$, respectively, are the incident angle over the FP and the related refracted angle in the FP. A $\theta$-dependent shift of the filter spectral band can be integrated at this level in $T_{\sigma}$.

We overlook, in this paper, the point spread function (PSF) of the microlens array, as it can be shown that at least $90 \%$ of the fringe visibility can be saved with a Nyquist sampling of the PSF. A convolution of the 2D NanoCarb image with the effective PSF could simulate this effect with a quite good accuracy.

Since we only consider homogeneous scene, this model can also be used to simulate NanoCarb L1a product, that is the partial 1D-interferogram at a given $\mathrm{iFoV}$ (given $\theta$ ).

Noise is added on this interferogram, taking into account photon noise (Poisson distribution) and additive read out noise (RON), with normal distribution.

\subsubsection{On thermal issues}

The $T$ dependency in Eq. (6) induced by temperature dependency of the optical index of silicon enables a thermal analysis of the system based on the model described above. This more advanced study is out of the scope of this paper, which focuses on the sensitivity of the concept for GHG measurement. However, preliminary investigations have shown that the interferometric envelope variation induced by a $0.1 \mathrm{~K}$ delta- $T$ is one magnitude smaller than the one induced by a $1 \mathrm{ppm}$ variation of $\mathrm{CO}_{2}$. Such a thermal regulation over a $0.1 \mathrm{~s}$ exposure is fully achievable with current cooling devices.

\subsection{Atmospheric model}

Terrestrial radiance $L_{\sigma}$ is simulated using the Standard US Model [20] for temperature and pressure profiles as well as atmospheric composition and concentration profiles. This model is implemented in the radiative transfer code LBLRTM [21], using the spectroscopic database HITRAN 
[22]. We simulate nadir terrestrial radiances with LBLRTM in the $1.6 \mu \mathrm{m}$ and $1.66 \mu \mathrm{m}$ band, taking into account fine spectroscopic effects such as line blending.

For this preliminary work, we assume a clear sky. $\mathrm{CO}_{2}$ and $\mathrm{CH}_{4}$ retrieval with input measurements of a joint aerosoldedicated instrument (SPEX [9]) will be implemented later in the framework of SCARBO. By focusing on the $1.6 \mu \mathrm{m}$ and $1.66 \mu \mathrm{m}$ band, we assume also that the atmospheric pressure is perfectly measured in the NanoCarb dedicated band.

\subsection{Analytical noise model}

We aim here at deriving an expression of the radiometric noise in ppm or ppb of the considered specie $X$ over the detector, to deliver a preliminary design of the instrument. A solution is to compare the required sensitivity for a targeted concentration variation, to the effective SNR over the fringe visibility measurement.

The required sensitivity $S_{\partial[X]}$ to measure a given concentration variation $\partial[X]$ of the specie $X$ can be expressed as the ratio of variation of the coherent flux $F_{X}$ in the targeted interferometric region:

$S_{\partial[X]}=\frac{\partial F_{X}}{\partial[X]} / F_{X}$

Replacing $V_{X}=F_{X} / \bar{N}_{\mathrm{ph}}$, the mean fringes contrast in the targeted Fourier region and $\bar{N}_{\text {ph }}$, the total mean number of photo-electron contributing to the coherent flux estimation, we obtain:

$S_{\partial[X]}=\frac{\partial V_{X}}{\partial[X]} / V_{X}+\frac{\partial \bar{N}_{\mathrm{ph}}}{\partial[X]} / \bar{N}_{\mathrm{ph}}$

We now introduce $S_{\mathrm{ph}, X}$, the effective sensitivity over the coherent flux, expressed as the inverse of the SNR over the coherent flux estimation $\hat{F}_{X}$ :

$S_{\mathrm{ph}, X}=1 / \operatorname{SNR}\left(\hat{F}_{X}\right)$,

given the mean interferometric intensities shown in Fig. 3, it is consistent to assume a photon-noise dominated regime. Under this condition, $\operatorname{SNR}\left(\hat{F}_{X}\right)$ is derived from [23]:

$\operatorname{SNR}\left(\hat{F}_{X}\right) \approx V_{X} \sqrt{\bar{N}_{\mathrm{ph}}}$

The ratio $S_{\mathrm{ph}, X} / S_{\partial[X]}$ gives a good appreciation of the NanoCarb radiometric sensitivity for the given concentration variation $\partial[X]$. Indeed, $\left(S_{\mathrm{ph}, X} / S_{\partial[X]}\right) \partial[X]$ is directly a noise over the concentration estimate in the same unit as $\partial[X]$. For example, a ratio of 0.5 for $\partial\left[\mathrm{CO}_{2}\right]=1 \mathrm{ppm}$ means a noise over L1b data product equivalent to $0.5 \mathrm{ppm}$.

Considering (8) and (10), we obtain:

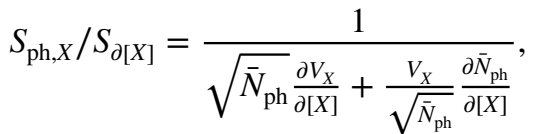

we can observe two terms at the denominator of the expression above:

- The left term shows as expected a statistical error improvement with the total number of photoelectrons as a function of the photon noise. Moreover, this term highlights the evolution of the NanoCarb sensitivity as a function of the contrast variation over the targeted interferometric regions. It requires a maximization of the interferometric Jacobian contrast, e.g., in the $5.6 \mathrm{~mm}$ OPD region for the $\mathrm{CO}_{2}$ (Fig. 3). Only the spectral filtering performed by the interferential filter permits such an optimization.

- The right term is a second order $\left(\sim 10^{-3}\right.$ compared to $\sim 10^{1}$ for the left term in the worst case). Nevertheless, it illustrates a potential downgrading of the sensitivity when an optimization of the Jacobian contrast decreases the total number of electrons in the targeted region. Thus, a joint trade-off must be carefully achieved to not degrade the fringe visibility when optimizing the spectral bandwidth.

On the maximum sensitivity interferometric area of the $\mathrm{CO}_{2}$ band, the expected $\partial V_{\mathrm{XCO}_{2}} /\left(\partial\left[\mathrm{CO}_{2}\right]=1 \mathrm{ppm}\right)$ is ranged around $10^{-4}$, calling for $\sim 10^{8}$ electron per iFoV for a target random error of $1 \mathrm{ppm}$ in a snapshot acquisition.

\subsubsection{Assumption over intensity level}

$\bar{N}_{\text {ph }}$ in expression Eq. (11) can state for the number of photoelectron contributing to the signal at L1a (snapshot frame) or L1b (final co-registered interferogram).

- On L1a snapshot partial interferogram: $\bar{N}_{\text {ph }}=\bar{N}_{\text {snap }} n_{\mathrm{FP}}$ is the mean total level of photoelectron contributing to the signal for a given $\mathrm{iFoV}$ in a single snapshot frame, with $n_{\mathrm{FP}}$ the number of Fabry-Perot for the considered spectral band.

- On L1b concatenated partial interferogram: As explained previously, the L1b data product is the coregistration of snapshot acquisitions while the $\mathrm{iFoV}$ shifts across the thumbnail during the orbit. Given $n_{\exp }$ the number of co-registered exposures for a given iFoV, we can express the total number of photoelectrons in L1b data products as:

$\bar{N}_{\text {ph }}=\bar{N}_{\text {snap }} n_{\text {FP }} n_{\text {exp }} ;[$ e/ifov $]$, 
Table 2 FPA characteristics used in NanoCarb radiometric study, based on NGP features [24]

\begin{tabular}{ll}
\hline FPA format & $1024 \times 1024$ \\
QE & 0.9 \\
Sensitivity range & $0.5-2.5 \mu \mathrm{m}$ \\
Pixel pitch & $15 \mu \mathrm{m}$ \\
Readout noise & $170 \mathrm{e}^{-}$ \\
Operating temperature & $170 \mathrm{~K}$ \\
Saturation level & $590 \mathrm{ke}^{-}$ \\
\hline
\end{tabular}

$n_{\text {exp }}$ depends on both the number of pixels per thumbnail side $N_{\mathrm{S}}$ and on acquisition frame rate. To permit signal co-registration, the minimum frame rate must be set to achieve an orbital brooming of one pixel between two exposures. Thus, the maximum number of co-registered exposures for one single $\mathrm{iFoV}$ is:

$\max \left\{n_{\exp }\right\}=N_{\mathrm{S}} \times$ broom $_{\text {pix }}$,

where broom $_{\text {pix }}$ is the orbital brooming expressed in pixel.

\section{Optimization of the spectral bands}

This section aims to illustrate the design strategy of the NanoCarb spectral bands, for SNR purposes. We expect to demonstrate the radiometric performances of NanoCarb and its potential for the SCARBO mission, expressed in terms of statistical error over the total column of $\mathrm{CO}_{2}$ or $\mathrm{CH}_{4}$ measurement, with our forward model.

\subsection{Investigated NanoCarb design}

We based this preliminary design over the state-of-the-art MCT SWIR FPA $1 \mathrm{k} \times 1 \mathrm{k}$ NGP [24], which has already been chosen for the MicroCarb mission [25] for instance. Relevant FPA characteristics are presented in Table 2. We nominally allocate one FPA per spectral band (1024 by 1024 pixels), as illustrated in Fig. 6.

We target a pixel sampling at ground of $1 \mathrm{~km}$, enabling a ground resolution of $2 \times 2 \mathrm{~km}^{2}$ with a Nyquist PSF sampling. A choice of $128 \times 128$ pixels per thumbnail provides a good trade-off between swath and number of interferometric samples, for a $1920 \mu \mathrm{m}$ FP and microlens pitch. Hence, we achieve a $128 \mathrm{~km}$ swath for an 8 by 8 Fabry-Perot interferometer array. The targeted effective acquisition frame rate is chosen for one pixel brooming per frame. We derive the maximum enabled exposure time from this frame rate. Table 3 summarizes these elements of design we consider for the radiometric study.

\subsection{Parametric optimization of the SNR}

With the basic design above, we present here an optimization of the NanoCarb $\mathrm{CO}_{2}$ and $\mathrm{CH}_{4}$ spectral bands, using synthetic interferograms, coupled with our radiometric performance model, and a model of 4-cavities narrowband filter. The optical transmission of the device is in range about $80 \%$. We assume a linear evolution of the absorbed power

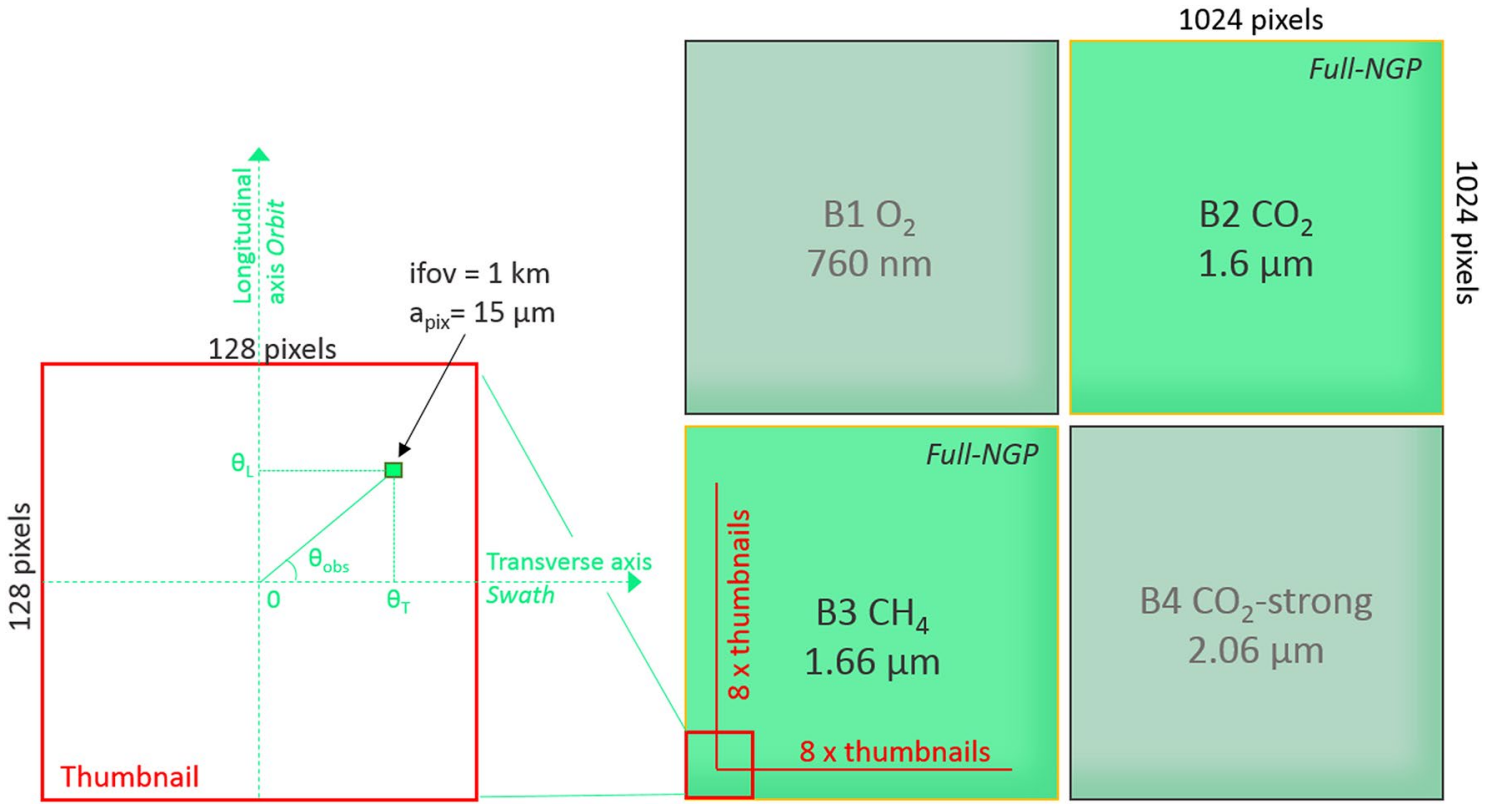

Fig. 6 NanoCarb allocated spectral bands and nominal investigated configuration of spatial sampling 
Table 3 Nominal NanoCarb band configuration for performance estimation

\begin{tabular}{ll}
\hline Altitude & $600 \mathrm{~km}$ \\
Frame rate & 1 -pixel brooming \\
Dedicated FPA size & $1024 \times 1024$ \\
Pixel per thumbnail side $N_{\mathrm{S}}$ & 128 \\
Number of Fabry-Perot and thumbnails $n_{\mathrm{FP}}$ & 64 \\
Pixel sampling at ground (iFoV) & $\mathbf{1 ~ k m}$ \\
Swath (fov) & $128 \mathrm{~km}$ \\
Maximum exposure time & $144.46 \mathrm{~ms}$ \\
\hline
\end{tabular}

with the concentration. This last point is well suited on the unsaturated $1.6 \mu \mathrm{m}$ and $1.66 \mu \mathrm{m}$ bands we consider here.

We vary the filter central wavenumber $\sigma_{0}$ over the spectral regions of interest, as well as its full width at half maximum (FWHM) from 1 to $100 \mathrm{~cm}^{-1}$. For each couple $\left\{\sigma_{0}\right.$; FWHM $\}$, we compute the single-iFoV snapshot radiometric sensitivity from a differential interferometric envelope $\partial V / \partial X$, with $\partial X$ a finite variation of concentration for the specie $X$. On this envelope, we search where the fringe visibility is maximum in the range of OPD corresponding to the Fourier signature of absorption line mean periodicity over the related spectral band.

Figure 7 shows the obtained map of sensitivity, expressed in $\mathrm{ppm}^{-1}$ and $\mathrm{ppb}^{-1}$ per iFoV and per frame, respectively, for $\mathrm{CO}_{2}$ and $\mathrm{CH}_{4}$.

- The red squares in the maps spot the optimal filter in terms of SNR.

- The contours highlight regions as a percentage of the optimal sensitivity.

- The partial derivatives in radiance for $\partial\left[\mathrm{CO}_{2}\right]=1 \mathrm{ppm}$ and $\partial\left[\mathrm{CH}_{4}\right]=10 \mathrm{ppb}$ are superimposed to the $\sigma_{0}$ scale, illustrating the evolution of the sensitivity with the periodic spectroscopic pattern.

- Some regions in the maps are aberrant, and set to zero. On the $\mathrm{CO}_{2}$ map: some parts of the region around $\sigma_{0}=6280 \mathrm{~cm}^{-1}$ enclosed by the $25 \%$ contour; on the $\mathrm{CH}_{4}$ map: the region around $\sigma_{0}=6000 \mathrm{~cm}^{-1}$. On these regions, the algorithm fails to find a signature on the targeted interferometric range. In the case of $\mathrm{CO}_{2}$, there are no $\mathrm{CO}_{2}$ absorption lines, and the interferogram is dominated by residual continuum shape. Concerning $\mathrm{CH}_{4}$, the band is dominated by saturated line group around $6000 \mathrm{~cm}^{-1}$.

$\mathrm{CO}_{2}$ : the line periodicity is small $\left(\sim 2 \mathrm{~cm}^{-1}\right)$ and presents some quite important jitters along the $\sigma_{0}$-axis. As a consequence, the maximal sensitivity evolves rapidly with a shift of spectral band, which is responsible for unstabilities of the relative $\mathrm{CO}_{2} / \mathrm{H}_{2} \mathrm{O}$ sensitivity in the $\mathrm{FoV}$ for the optimal red point $\left\{\sigma_{0}=6216 \mathrm{~cm}^{-1} ; \mathrm{FWHM}=17 \mathrm{~cm}^{-1}\right\}$. The symmetric optimal filter $\left\{\sigma_{0}=6336.3 \mathrm{~cm}^{-1} ; \mathrm{FWHM}=17 \mathrm{~cm}^{-1}\right\}$ is also rejected due to a huge amount of water on this region. The finally chosen filter $\left\{\sigma_{0}=6213 \mathrm{~cm}^{-1}\right.$; FWHM $\left.=24 \mathrm{~cm}^{-1}\right\}$ is a trade-off between $\mathrm{CO}_{2} / \mathrm{H}_{2} \mathrm{O}$ sensitivity, SNR, and stability in the FoV.

$\mathbf{C H}_{4}$ : the periodicity of the $\mathrm{CH}_{4}$ absorption line is large $\left(\sim 12 \mathrm{~cm}^{-1}\right)$. Consequently, the optimal filter $\left\{\sigma_{0}=6078 \mathrm{~cm}^{-1}\right.$; FWHM $\left.=69 \mathrm{~cm}^{-1}\right\}$ is wide, and the sensitivity slowly revolves around this optimal point, which is convenient to design the narrowband filter. Hence, despite of the large amount of water on this band, some interesting interferometric water-free regions, stable in the FoV, can be found as we will see in next section. Thus, the red spotted filter parameters are chosen for B3.

Table 4 summarizes the selected B2 and B 3 bands.

\subsection{Extrapolation of the noise over $\mathrm{CO}_{2}$ and $\mathrm{CH}_{4}$ measurements on $\mathrm{L} 1 \mathrm{~b}$ data product}

The last sub-section illustrated the strategy to design the spectral bands of NanoCarb. Given the two retained B2 and B3 bands, we aim at extrapolating here the intrinsic radiometric sensitivity of the instrument at L1b level.

We plot in Fig. 8 the statistical error on $\mathrm{CO}_{2}$ or $\mathrm{CH}_{4}$ concentration measurement as expressed in Eq. (11) as a function of the percentage of maximum co-registered snapshot acquisitions $\max \left\{n_{\exp }\right\}$. We assume here that all the interferometric samples of NanoCarb are located on the maximum $\mathrm{CO}_{2}$ or $\mathrm{CH}_{4}$ sensitivity area of each band derived from the previous study. We investigate four observation scenarios: 1 and $1.5 \mathrm{~km}$ of ground pixel sampling (ground resolution respectively $2 \times 2$ and $3 \times 3 \mathrm{~km}^{2}$ ), and a terrestrial albedo of 0.05 and 0.2 with a solar zenithal angle of $55^{\circ}$ within clear sky.

\subsubsection{Statistical error on $\mathrm{CO}_{2}$ measurement}

The exploitation of all the available snapshot acquisitions largely fulfills sub-ppm noise on $\mathrm{CO}_{2}$ measurement, in all the considered configurations, and observation conditions. The performances are even below $0.5 \mathrm{ppm}$ in the more favorable observation conditions (albedo $>0.2$ ). Hence, the use of only $50 \%$ of the available snapshot acquisitions allows again to reach a sub-ppm noise. This is a benefit to mitigate interpolation issues between the different frames caused, for example, by platform dis-pointing or jitter, as well as cloud effects.

\subsubsection{Statistical error on $\mathrm{CH} 4$ measurement}

The 10-ppb sensitivity target is reached too in all observation conditions, with the co-registration of at least $50 \%$ of 

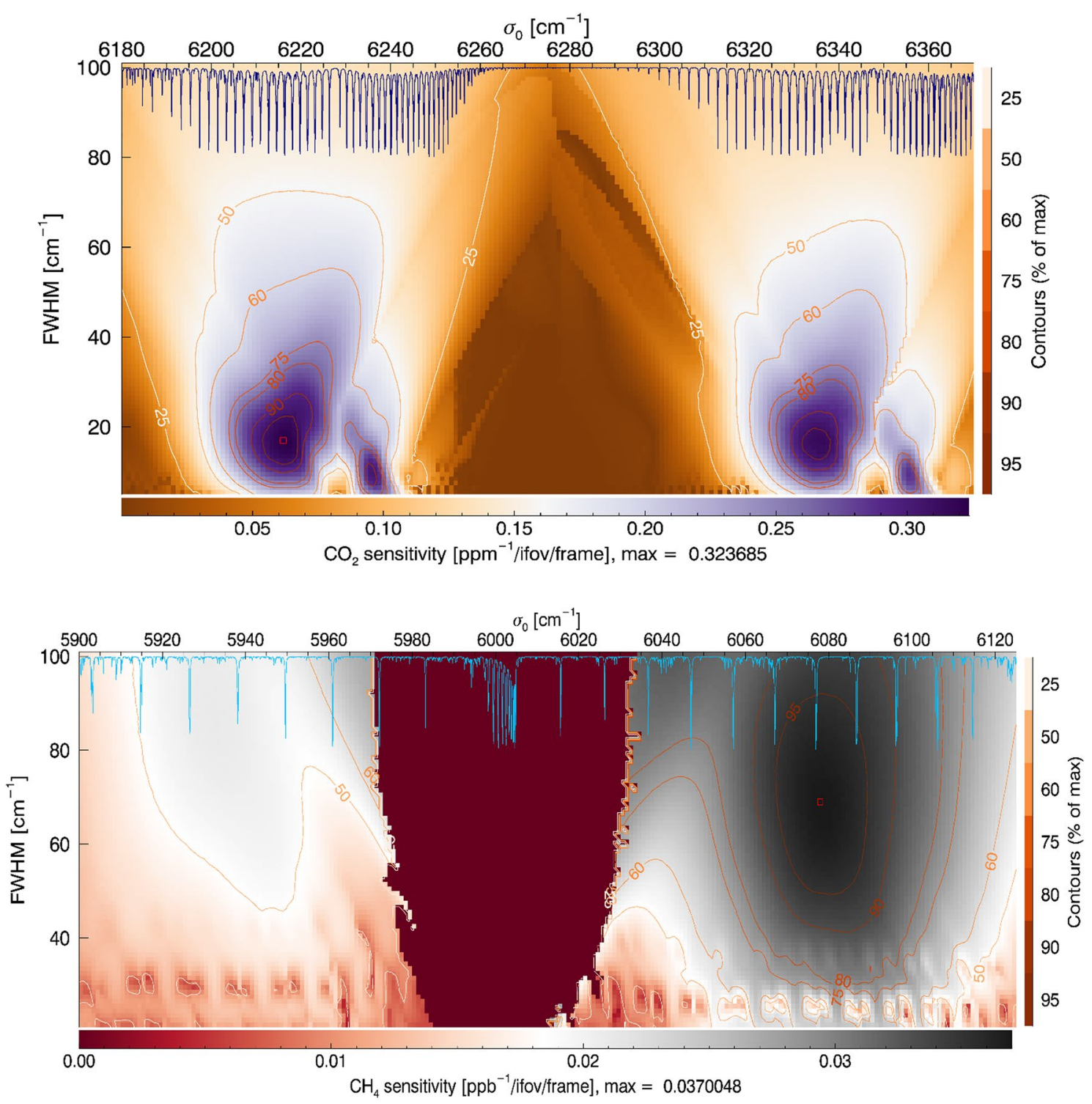

Fig. 7 Sensitivity evolutions for $\mathrm{CO}_{2}$ (top) and $\mathrm{CH}_{4}$ (bottom) at maximum OPD as a function of the interferential filter parameters: central wavenumber $\sigma_{0}$ ( $x$-axis) and FWHM ( $y$-axis). To the top of each map is superimposed the differential spectrum, respectively, for a finite

Table 4 Selected filter for B2 and B3 bands and relevant snapshot sensitivity, given a solar zenithal angle of $55^{\circ}$ and an albedo of 0.2

\begin{tabular}{llll}
\hline Band & Filter & & $\begin{array}{l}\text { Max. snapshot } \\
\text { sensitivity/iFoV }\end{array}$ \\
\cline { 2 - 3 } & $\sigma_{0}\left(\mathrm{~cm}^{-1}\right)$ & $\begin{array}{l}\text { FWHM } \\
\left(\mathrm{cm}^{-1}\right)\end{array}$ & \\
\hline $\mathrm{B} 2-\mathrm{CO}_{2}-1.60 \mu \mathrm{m}$ & 6213 & 24 & $3.10 \mathrm{ppm}$ \\
$\mathrm{B} 3-\mathrm{CH}_{4}-1.66 \mu \mathrm{m}$ & 6079 & 69 & $27.02 \mathrm{ppb}$ \\
\hline
\end{tabular}

the available frames. We can expect a noise around $5 \mathrm{ppb}$ in the worst observation conditions (albedo $=0.05$ ), by coregistering all the available frames. variation of $1 \mathrm{ppm}$ of $\mathrm{CO}_{2}$ and $10 \mathrm{ppb}$ of $\mathrm{CH}_{4}$. The contours highlight evolutions of the sensitivity as a percentage of the optimal filter (red square)

In conclusion, within optimal instrumental conditions (no instrumental defects, photon noise only, perfect knowledge of the other geophysical parameters):

- $\quad 0.2-1 \mathrm{ppm}$ target for $\mathrm{CO}_{2}$ noise is reached with a nominal allocation of one NGP for the $1.6 \mu \mathrm{m}$ band.

- 1-5 ppb for $\mathrm{CH}_{4}$ noise is reached in the same conditions.

- These good intrinsic performances enable (1) a decrease of the exposure time up to a factor 4 to mitigate pointing blurring effects, (2) the allocation of samples to other dedicated areas of the interferogram, for example, to jointly measure water. 

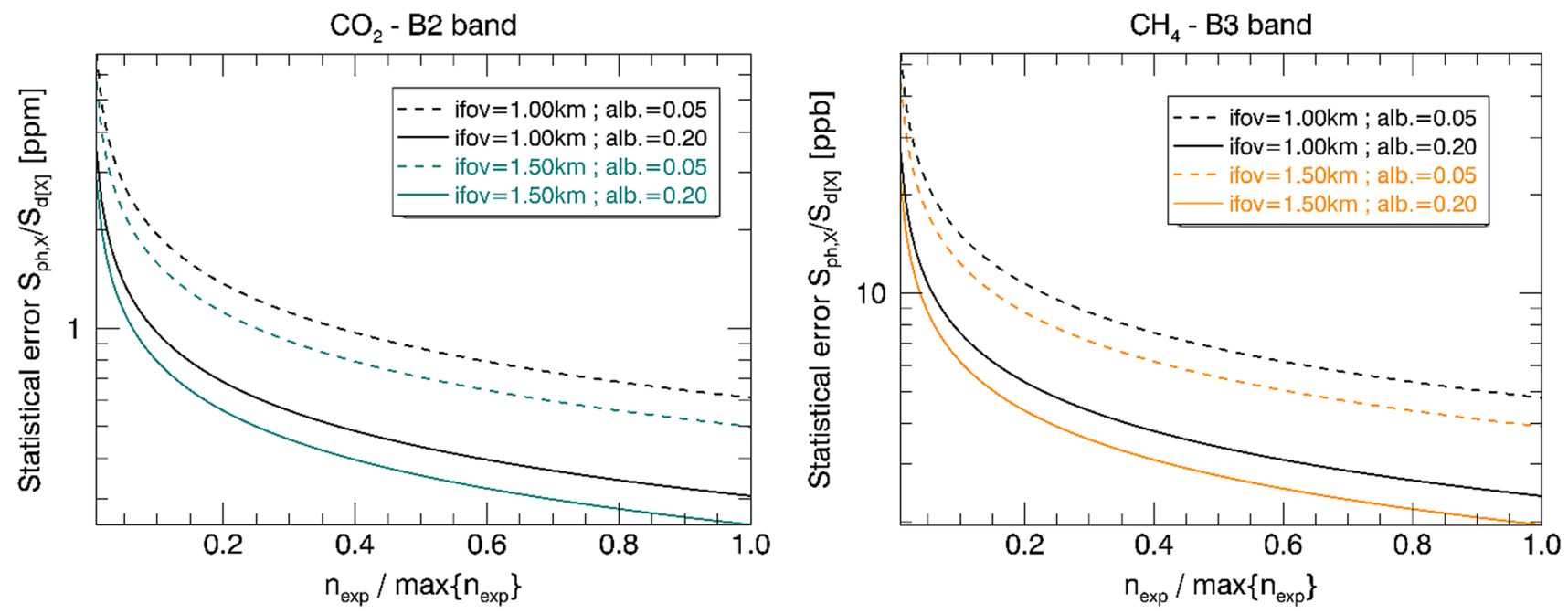

Fig. 8 Evolution of the NanoCarb statistical error on $\mathrm{CO}_{2}$ and $\mathrm{CH}_{4}$ measurements as a function of the number of co-registered exposures. On $x$-axis, one means the use of the total number of available frames when the iFoV shifts across the thumbnail, while zero is the snapshot mode

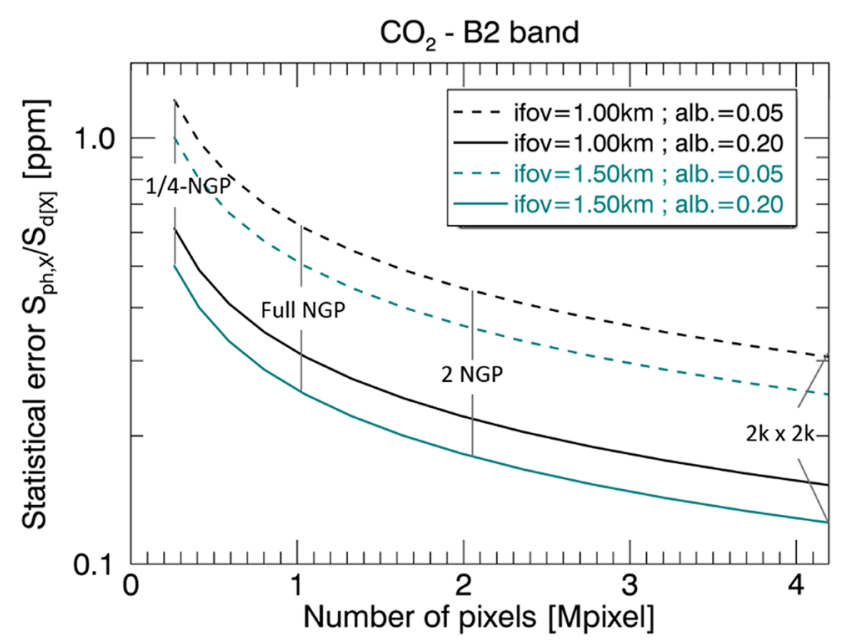

Fig. 9 Evolution of the noise on L1b as a function of the total number of pixels over the FPA. The statistical error is given for a co-registration of all the available frames $\max \left\{n_{\exp }\right\}$. The number of pixels per thumbnail and the pixel pitch are unchanged. Thus, the number of

To go further, an interesting feature of the NanoCarb concept is the dependence of the noise on L1b data to the number of pixels used on the considered spectral band: the number of thumbnails (or FP), $n_{\mathrm{FP}}$, can be easily increased by enlarging the FPA area dedicated to the spectral band, any other parameters fixed. Consequently, $\bar{N}_{\text {ph }}$ will increase linearly with the size of the dedicated FPA area. Figure 9 shows the noise trends as a function of the number of dedicated pixels, in the case we co-register all the available frames.

This optimization of the NanoCarb radiometric performances is done with a very faint evolution of the instrumental complexity. As the number of pixels increases for

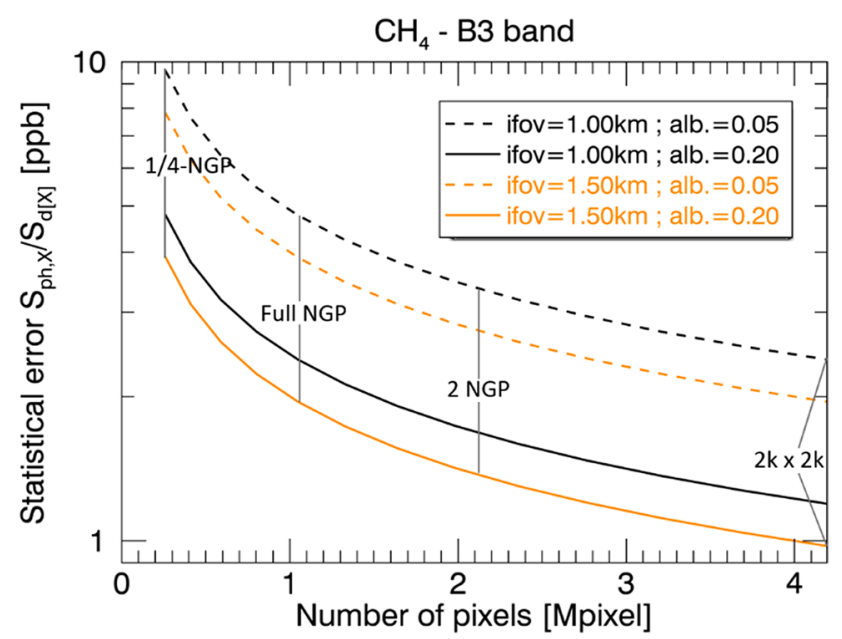

co-registered exposures is kept constant, as well as the exposure time. The $x$-axis is equivalent to an increase in the number of thumbnails, and thus, to the number of interferometric samples

the totality of the spectrometer, we are confident to a linear increase of the volume of the optical part of NanoCarb (from the objective to the FPA), driven by the size of the sensitive area on the detector. As an example, if we evaluate a volume of $250 \mathrm{~cm}^{3}$ with one NGP, the volume with a next generation $2 \mathrm{k} 2 \mathrm{k}$ NGP-like [26] will be approximately increased by a factor of eight. Weight and full thermal regulation must be treated as such, which is a real advantage compared to dispersive spectrometry techniques and scanning Fourier-transform spectrometers.

We demonstrated here the intrinsic radiometric capabilities of the NanoCarb concept for $\mathrm{CO}_{2}$ and $\mathrm{CH}_{4}$, in a 
Table 5 Geophysical variables to be retrieved from NanoCarb measurement, and impact over the different bands. In green - the dedicated spectral bands when occurred

\begin{tabular}{|l|cccc|}
\hline \multirow{2}{*}{\multicolumn{1}{|c|}{ Geophysical variable }} & \multicolumn{4}{c|}{ Sensitivity } \\
\cline { 2 - 6 } & $B 1$ & $B 2$ & $B 3$ & $B 4$ \\
\hline $\mathrm{CO}_{2}$ profile & $\times$ & Yes & $\times$ & Yes \\
\hline $\mathrm{CH}_{4}$ profile & $\times$ & $\times$ & Yes & $\times$ \\
\hline $\mathrm{H}_{2} \mathrm{O}$ profile & $\times$ & Yes & Yes & Yes \\
\hline Surface pressure & Yes & Yes & Yes & Yes \\
\hline Albedo & Yes & Yes & Yes & Yes \\
\hline Clouds and aerosols optical depth & Yes & Yes & Yes & Yes \\
\hline
\end{tabular}

forward approach. Nevertheless, this study does not study any impact of the instrumental or geophysical biases, and does not allow consequently to accurately choose the interferometric samples.

\section{Design of the Fabry-Perot micro-interferometer array}

Previous section focused on band optimization for SNR purposes. We develop and illustrate here a proper strategy to mitigate bias impacts over $\mathrm{CO}_{2}$ or $\mathrm{CH}_{4}$ measurement, by designing the micro-FP interferometer array and choosing the OPDs to sample. We mainly present water-related issues as justified in a first sub-section. A second sub-section presents a forward exploration of the information content of the interferogram, as the last one introduces an inverse approach to estimate the performances and generalize this optimization.

\subsection{Biases over B2 and B3 bands and geophysical variables}

The main geophysical variables to be retrieved, which affect the NanoCarb measurement over the previously considered spectral bands, are summarized in Table 5 .

These geophysical parameters potentially bias the measurement of averaged $\mathrm{CO}_{2}$ and $\mathrm{CH}_{4}$ dry column, and must be addressed in the design of the micro-interferometer array. Nevertheless, at this preliminary level of design, we focus in this paper only on B2 and B3 bands:

- Clouds and aerosols issues, monitored on B1 and B4 bands, are consequently not considered despite their huge impact over $\mathrm{CO}_{2}$ and $\mathrm{CH}_{4}$ measurement. We assume a perfect joint measurement by SPEX, similar to clear sky assumption. An upcoming study will be led to assess the un-bias capability of the SPEX instrument over Nano-
Carb measurement, with a realistic model of performances.

- We assume a perfect joint estimation of surface pressure from B1 band.

- The albedo estimation accuracy relies on the radiometric calibration capability of the device, and has not been yet studied. Works based on experimental characterization are in progress to evaluate FPA mis-calibration impact over our signal knowledge. A discussion about it is proposed in paragraph 5.4.

- Water is one of the main interferants affecting B2 and B3 bands, and is consequently treated in this preliminary design of micro-interferometer array.

We present below a simple study of the impact of a misestimation of the total column of $\mathrm{H}_{2} \mathrm{O}$ over the averaged column of $\mathrm{CO}_{2}$ retrieval. The goal is to derive an estimation of the required accuracy we have to reach, for illustrative purposes. The retrieval algorithm is a simple least mean square method based on a Levenberg-Marquardt algorithm. Its specificity is to work with partial concatenated L1b interferograms, without intermediate radiance spectrum retrieval.

We target the $\mathrm{B} 25.6 \mathrm{~mm}$ area of the interferogram where the sensitivity to $\mathrm{CO}_{2}$ is optimal. All the $64 \mathrm{FP}$ are dedicated to the sampling of this region. Apart from the bias on $\mathrm{H}_{2} \mathrm{O}$, the retrieval L1b model is perfect, and the simulated data are noiseless. The $\mathrm{CO}_{2}$ initial guess for the retrieval is about $95 \%$ of the data value $(400 \mathrm{ppm})$. Figure 10 presents the evolution of the absolute accuracy over $\mathrm{CO}_{2}$ estimate with respect to the bias on water. We notice a strong impact of a water misestimation, because $10 \%$ error on water induces a bias greater than $1 \mathrm{ppm}$ over the $\mathrm{CO}_{2}$, while $1 \%$ leads to a bias greater than $0.1 \mathrm{ppm}$.

This observation was well expected if we consider the $\mathrm{CO}_{2}$ interferogram as in Fig. 3-left: the partial derivative for $1 \mathrm{ppm}$ of $\mathrm{CO}_{2}$ is comparable in flux to the one for $10 \%$ of water in the $5.6 \mathrm{~mm}$ region. Therefore, both $\mathrm{H}_{2} \mathrm{O}$ and $\mathrm{CO}_{2}$ have a significant and comparable impact over the fringe intensity. With similar considerations, the impact of water over $\mathrm{CH}_{4}$ band is greater by a factor 10 at least.

This result justifies the need to refine the NanoCarb design to mitigate the water impact on B2 and B3 bands. Especially, we will consider in the next sub-section the selection of several dedicated interferometric regions in a forward approach.

\subsection{Design for water mitigation with forward model}

The forward model of NanoCarb is implemented here to search for interferometric regions in $\mathrm{B} 2$ and $\mathrm{B} 3$ bands where sensitivity to $\mathrm{CO}_{2}$ and $\mathrm{CH}_{4}$ is optimal related to water and instrumental issues, especially the spectral band 


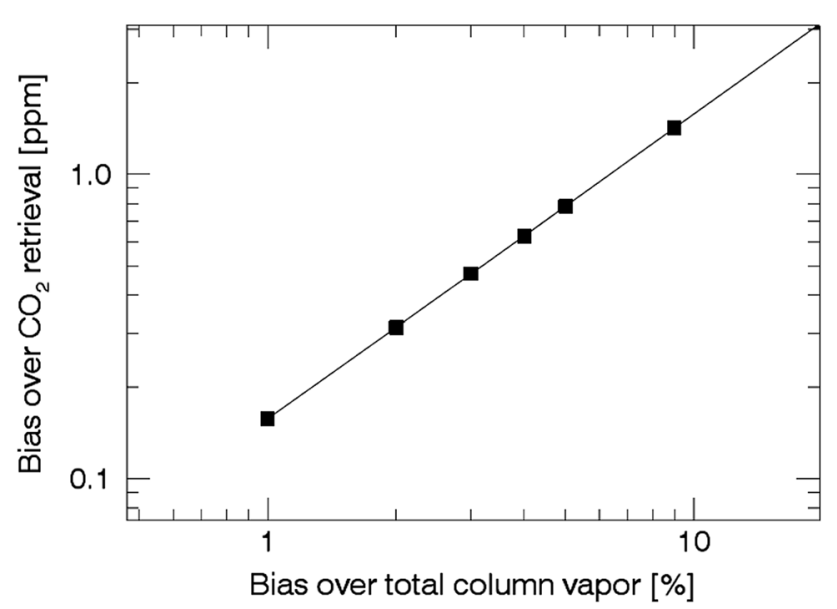

Fig. 10 Evolution of the bias over total column of $\mathrm{CO}_{2}$ retrieval as a function of a misestimation of the total column of $\mathrm{H}_{2} \mathrm{O}$. The maximum $\mathrm{CO}_{2}$ sensitivity region of the interferogram for the chosen filter is targeted

shift in the FoV of the filter, responsible for interferometric signature variabilities and instabilities.

Figure 11 shows central wavenumber shift in the FoV of a thumbnail. For the investigated design, the spectral shift is quite similar in B2 and B3 bands, with a maximum of $+9.5 \mathrm{~cm}^{-1}$ on the corners, and $+5 \mathrm{~cm}^{-1}$ at maximum on axis.

Full interferograms are computed over a relevant range of OPD. Then the envelope variations $\partial V / \partial X$ are derived, with $X$, respectively: a $1 \mathrm{ppm} \mathrm{CO}_{2}$ finite variation, a $1 \mathrm{ppb}$ $\mathrm{CH}_{4}$ variation, and a $10 \% \mathrm{H}_{2} \mathrm{O}$ variation. These envelopes are plotted on Figs. 12 and 13, for each pixel of a half thumbnail along the longitudinal axis (direction of signal co-registration) and for four $\mathrm{iFoV}$ on the swath (transverse axis) as represented by multi-colored arrows in Fig. 11.

On Figs. 12 and 13, the superimposed blue curves show the evolution of the water interferometric signature along the longitudinal axis, while the brown-white-green curves state for $\mathrm{CO}_{2}$ on B2 on Fig. 12 and $\mathrm{CH}_{4}$ on B3 on Fig. 13. Each graph $(\mathrm{a}-\mathrm{d})$ on both Figs. 12 and 13 represents one among the four selected $\mathrm{iFoV}$ on the swath.

$\mathrm{CO}_{2}$ on B2 Fig. 12: The maximum of sensitivity for $\mathrm{CO}_{2}$ at $5.6 \mathrm{~mm}$ seems stable in the $\mathrm{FoV}$ even for the most extreme $\mathrm{iFoV}$ of the swath. Nevertheless, this interferometric region is also sensitive to water in quite important proportions (typically $50 \%$ of $\mathrm{CO}_{2}$ visibility), calling for dedicated $\mathrm{H}_{2} \mathrm{O}$ disjoint region. The $1.2 \mathrm{~mm}$ water region is rejected due to huge sensitivity variations in the FoV. On the contrary, the $2.4 \mathrm{~mm}$ water-dominated region is stable in the FoV while the sensitivity to $\mathrm{CO}_{2}$ is almost null. As a conclusion, the $\mathrm{CO}_{2}$ water-biased $5.6 \mathrm{~mm}$ region and the water $\mathrm{CO}_{2}$-free $2.4 \mathrm{~mm}$ region are selected to guarantee an unbiased measurement of $\mathrm{CO}_{2}$ concentration.

$\mathrm{CO}_{2}$ on $\mathrm{B} 2$ has a harmonic signature at $11.2 \mathrm{~mm}$ of OPD that we did not consider here. Indeed, such a high OPD induces high frequency FP ring pattern and consequently potential pixel sampling issue. This consideration is related to the interferometric transposition of the Jacquinot criterion about spectral resolution and sampling.

$\mathbf{C H}_{4}$ on B3 Fig. 13: The $\mathrm{CH}_{4}$ signature presents several harmonic regions every $1.05 \mathrm{~mm}$ of OPD, and thus as many sensitive areas. Nevertheless, only the first two have fringes in a favorable proportion of sensitivity $\mathrm{CH}_{4} / \mathrm{H}_{2} \mathrm{O}$, and we limit the study to the $2.5 \mathrm{~mm}$-wide plotted regions. $\mathrm{CH}_{4}$ signature is very stable in the $\mathrm{FoV}$, as well as several
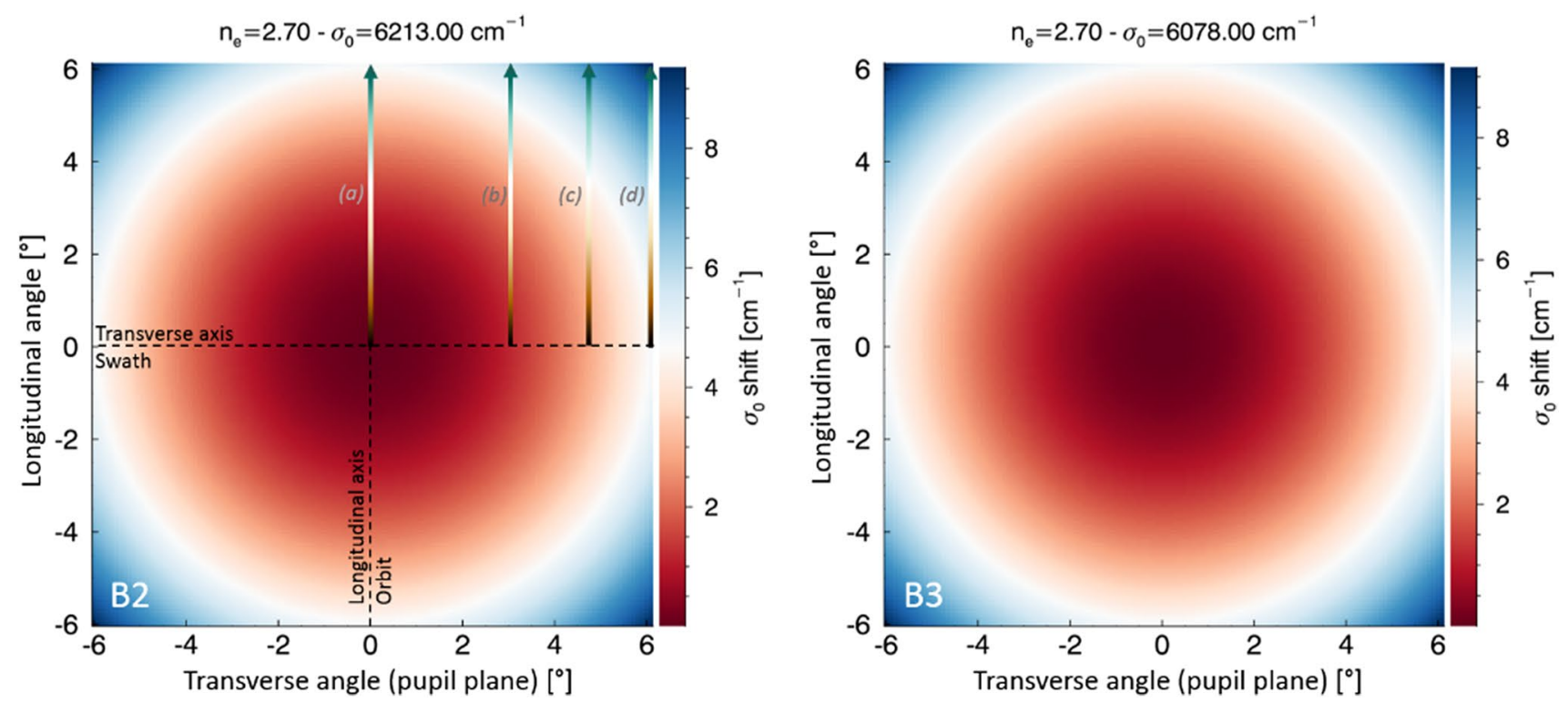

Fig. 11 Filter central wavenumber shift in the FoV of a thumbnail for B2 (left) and B3 (right). $n_{\mathrm{e}}$ is the effective index of the interferential filter 

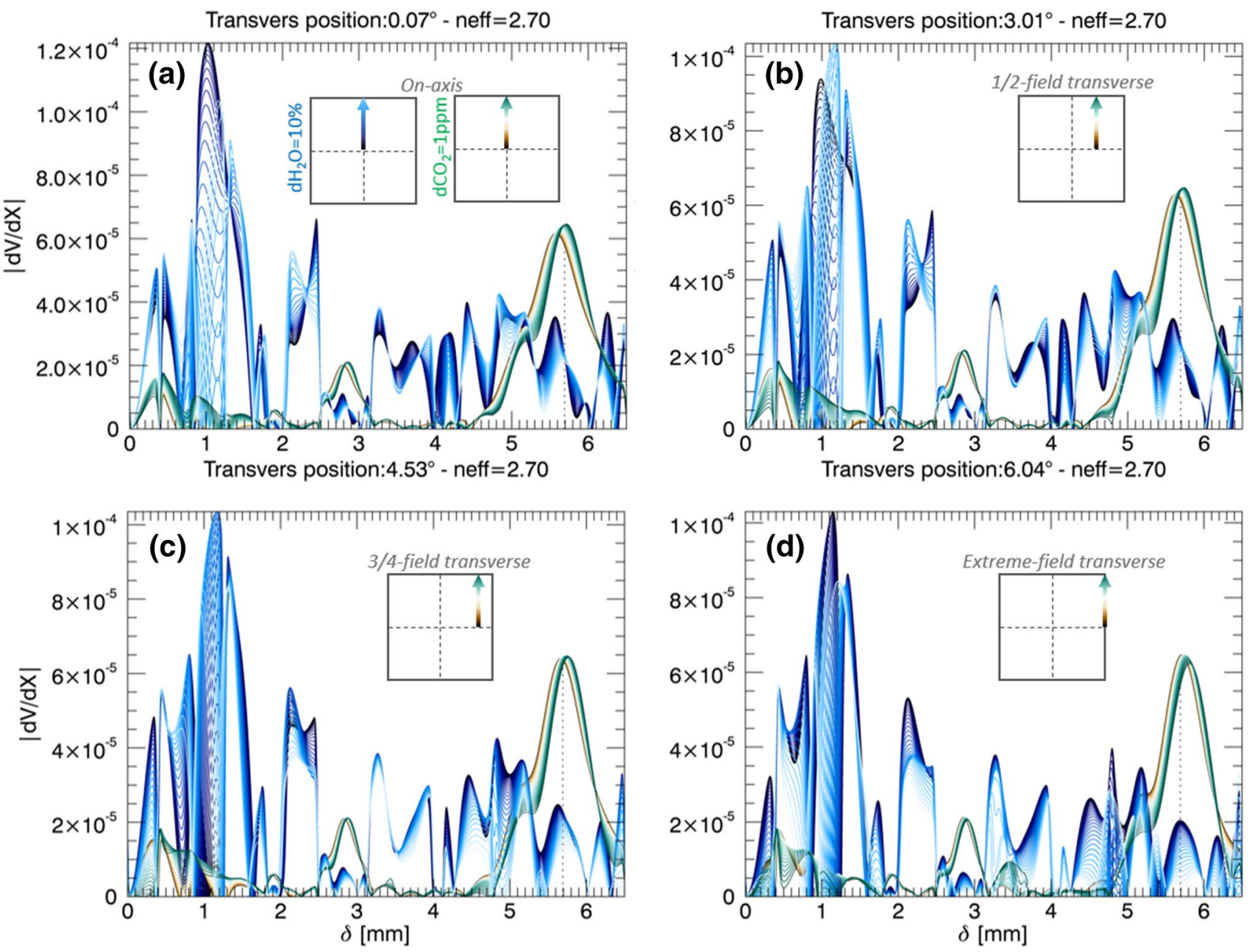

Fig. 12 Evolution of the $\mathrm{CO}_{2}$ interferometric signature-envelopealong the longitudinal axis (brown-white-green superimposed curves), and of the water (blue superimposed curves). a iFoV at the

center of the swath; $\mathbf{b}$ iFoV at the half-swath; $\mathbf{c}$ iFoV at the $3 / 4$ of the total swath; $\mathbf{d}$ extreme $\mathrm{iFoV}$ on the swath

water areas such as at $0.9 \mathrm{~mm}, 1.25 \mathrm{~mm}$, and $2.35 \mathrm{~mm}$. The maximum sensitivity of $\mathrm{CH}_{4}$ at $1.05 \mathrm{~mm}$ seems to be free of water. Additional samples placed on the second $\mathrm{CH}_{4}$ harmonic could provide also a measurement of a more varied kind of information, for instance about vertical profile.

\subsection{Illustration of $\mathrm{CO}_{2}$ and water retrieval with two OPD areas}

To illustrate the bias reduction provided by the choice of specific OPD areas in the same spectral band, we present in Fig. 14 a preliminary retrieval result from a L1b B2 measurement vector. This vector is composed by eight water-dedicated samples around $2.4 \mathrm{~mm}$ of OPD, and 54 $\mathrm{CO}_{2}$-dedicated samples around $5.6 \mathrm{~mm}$ of OPD. The NanoCarb configuration is the one defined in Table 3 , and the filter is the B2 filter defined in Table 4. For this retrieval run, we consider a perfect inverse model, noiseless data, and a guess concentration of $90 \%$ of the real value for the total column of $\mathrm{CO}_{2}$ and $\mathrm{H}_{2} \mathrm{O}$. The retrieval algorithm is the same as used in sub-Sect. 5.1.

Despite a slow convergence of the water estimation, this very interesting result shows a quick convergence of the $\mathrm{CO}_{2}$ column retrieval toward a numerically limited fraction of the real value. It seems that the chosen partial sampling of the interferogram permits in this case a robust degeneracy removing of water over the $\mathrm{CO}_{2}$ retrieval.

The next sub-section introduces some leads to improve this design as well as assess its performances.

\subsection{Discussion about design optimization and performances assessment}

We illustrated previously the forward strategy to design the NanoCarb $\mu$ FP array considering one of the main interferant over B2 and B3 bands, allowing to establish a preliminary 

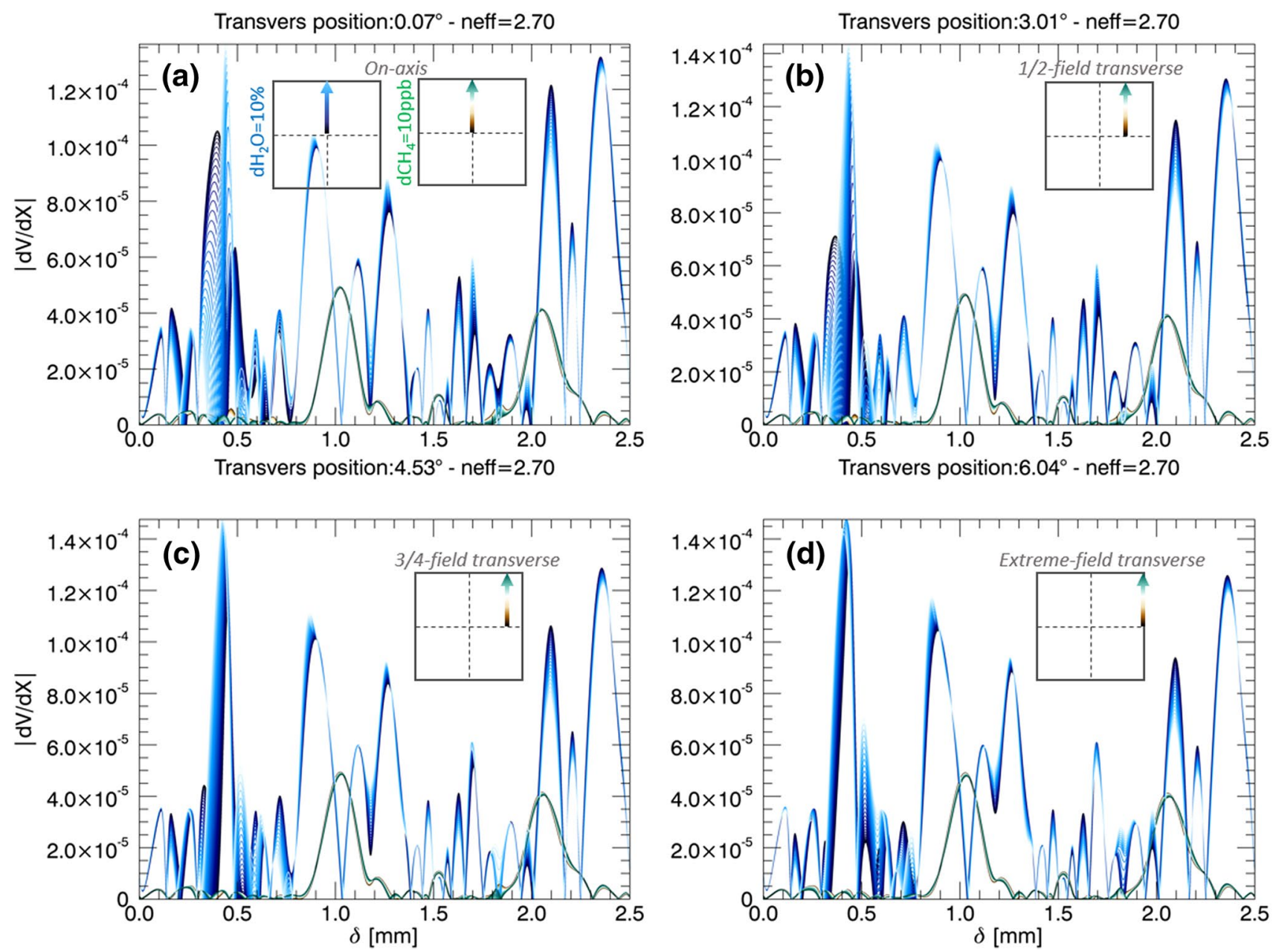

Fig. 13 Evolution of the $\mathrm{CH}_{4}$ interferometric signature-envelopealong the longitudinal axis (brown-white-green superimposed curves), and of the water (blue superimposed curves). a iFoV at the

center of the swath; $\mathbf{b}$ iFoV at the half-swath; $\mathbf{c}$ iFoV at the $3 / 4$ of the total swath; $\mathbf{d}$ extreme iFoV on the swath

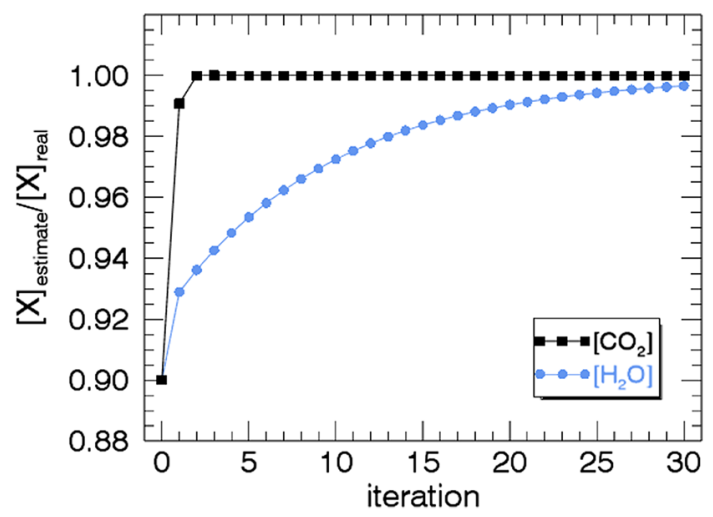

Fig. 14 Retrieval convergence of a joint estimation of $\mathrm{CO}_{2}$ and $\mathrm{H}_{2} \mathrm{O}$ columns on the $1.6 \mu \mathrm{m}$ band, by sampling two dedicated disjoint regions on the interferogram, one for water and the other one for $\mathrm{CO}_{2}$

design of the instrument. However, some questions remain about OPD choice, which cannot be assessed with this model:

- Generalization of the approach to all the geophysical variables described in Table 5, as well as instrumental biases.

- Number of allocated FPs (samples) per identified region.

- Sampling strategy: (1) multi-point sampling of the interferometric envelope, calling for multiple disjoint regions, or (2) concentration of all the samples on some regions illustrated in the previous sub-section. While the concentration of samples at maximum sensitivity areas ensures a maximum SNR, the variety of measured information is very poor, potentially responsible for important sensitivity to other geophysical biases or vertical-induced profile effects. 
Table 6 Main considered instrumental issues for a NanoCarb-based payload, related impact over the performances, and assumed criticality for the final data quality considering knowing solutions

\begin{tabular}{|c|c|c|c|}
\hline & Impact & Solution & Criticality \\
\hline Spectral response calibration & High & OCT scan or spectral scan of each narrowband & Low \\
\hline Radiometric calibration & High & To be established & High \\
\hline Detector PRNU & High & Inflight calibration protocol has to be addressed & Low/high \\
\hline Parasitic/stray light & High & $\begin{array}{l}\text { High criticality known for many space instruments with a similar performance aim but } \\
\text { a different concept (e.g., dispersive spectrometer). }\end{array}$ & High \\
\hline $\begin{array}{l}\text { Filter thermal stability } \\
\text { Interferometer thermal stability }\end{array}$ & High & $\sim 0.1-1 \mathrm{~K}$ thermal regulation & Low \\
\hline Platform stability & Low & Specification to be determined & Low \\
\hline Component behavior in space & Unknown & $\begin{array}{l}\text { Radiation resistance of silicon-based components, multi-layer processed surfaces, } \\
\text { detector aging, etc., has to be addressed }\end{array}$ & Unknown \\
\hline
\end{tabular}

- Validation of the chosen interferometric samples in terms of reached absolute accuracy on L2 data product (systematic biases).

\subsubsection{Generalized bias mitigation strategy for OPD choice}

A simple way to take into account all the geophysical variables in the design of NanoCarb interferometers could be based on a retrieval model coupled with a Monte Carlo exploration of instrumental parameter space (position and number of samples, number of regions, etc.). A stray forward would be:

1. Fix the NanoCarb key features, for instance number of pixels, number of Fabry-Perot, FoV, etc.

2. Fix the spectral bands by SNR forward analysis as presented in the previous section.

3. Forward analysis of sensitivity of the interference domain as presented in sub-Sect. 5.2. The aim is to identify consistent interferometric regions and consequently restrain OPD range of analysis, for both all bands and geophysical parameters. A Bayesian approach of the information could be also considered as described in Sect. 2.5 of [11].

4. Multi-variable retrieval on L1b data for $n$ sets of $n_{\mathrm{FP}}$ samples chosen by Monte Carlo in the restrained parameter space.

This method potentially enables to derive performance trends as a function of the number of allocated samples per identified region as well as the distribution of regions along the interferometric envelope.

\subsubsection{Instrumental issues}

Instrumental issues are just starting to be addressed in the SCARBO framework. Table 6 gathers some of them identified for NanoCarb, for discussion purposes.
Both spectral response and radiometric calibration of the instrument are critical to retrieve radiometrically calibrated L1a, and then L1b interferograms from L0 raw images. Even if the spectral response characterization of the instrument does not constitute a stumbling block by using either interferometric techniques such as optical coherence tomography $[18,19]$ or a spectral scan, efforts have to be provided to ensure a radiometrically calibrated device, with an accuracy to be determined. This latter may have a critical impact to disentangle between albedo variations over the scene and aerosol-induced extinction. Moreover, questions remain about calibration stability in time and in-flight capabilities of re-calibration with the considered $50 \mathrm{~kg}$-class platform.

The Photo-Response Non-Uniformity (PRNU) of the detector and more exactly the residual fixed-pattern noise (RFPN) is related to the radiometric issue and must be treated as relevant, and its impact may be very significant with a Fourier-transform spectrometer [27].

Also related to radiometric calibration, and with a potential huge impact, is the issue of parasitic and stray light. Indeed, it has been observed in another FP-based space instrument such as GHGsat-D "CLAIRE" [28] that stray light may be a critical point for the performances. A snapshot static Fourier-transform spectrometer such as NanoCarb is potentially more sensitive to temporal stray light variation compared with push broom or scanning spectrometers, because each interferometric channel and iFoV are associated with one different pixel of the detector.

On the other hand, as introduced previously in this paper (sub-Sect. 3.1), thermal issue does not seem to be a hurdle to the instrument operability, since a $0.1-1 \mathrm{~K}$ thermal stability enables to avoid impacts over the measurement.

Platform stability and component behavior in space will be addressed later, as the efforts are currently focused on the demonstration of the NanoCarb concept feasibility at a quite early level. 
Laboratory prototypes are being integrated to address some of these issues, as well as an air-borne prototype is also planned for in-flight demonstration of the NanoCarb performances in a relevant environment.

\subsubsection{Systematic performances assessment}

The performance assessment of NanoCarb is an iterative work in progress in the H2020 SCARBO framework. A Bayesian optimal estimation of $\mathrm{CO}_{2}$ and $\mathrm{CH}_{4}$ averaged dry column is assessed from the presented model of NanoCarb for a complete set of atmospheres and many observation scenarios. The followed methodology has been applied for $\mathrm{CO}_{2} \mathrm{M}$ error parametrization [29]. This study, done with the Laboratoire de Météorologie Dynamique (LMD), ${ }^{2}$ will provide both expected random error of NanoCarb for $\mathrm{CO}_{2}$ and $\mathrm{CH}_{4}$ at $\mathrm{L} 2$ (to be compared with the noise at $\mathrm{L} 1 \mathrm{~b}$ presented in this paper), and systematic error. The latter will enable to validate the presented OPD choice and geophysical bias mitigation, or to refine it if relevant.

\section{Conclusion}

NanoCarb is an original concept of imaging spectrometer combining the use of innovative interferometric components and an unusual partial interferogram sampling technique. These two combined features are investigated in the SCARBO project to assess the feasibility of a constellation based on such miniaturized payloads to monitor the anthropogenic GHG emissions with a daily revisit and a global coverage.

In a forward approach, we showed the radiometric capabilities of this concept to measure $\mathrm{CO}_{2}$ and $\mathrm{CH}_{4}$ in the nearinfrared, through an optimal design of the narrowband filters. The expected theoretical and intrinsic performances, in terms of statistical error over measurement of the averaged column of $\mathrm{CO}_{2}$ and $\mathrm{CH}_{4}$, are comparable to other dedicated space missions. Then we addressed the design of the interferometer, to manage geophysical biases. The design strategy has been illustrated for water mitigation. For instance, a significant theoretical decrease in water-induced bias compared to $\mathrm{CO}_{2}$ measurement can be expected using an $8 \times 8$ interferometer array with 8 water-dedicated and 54 $\mathrm{CO}_{2}$-dedicated Fabry-Perot.

The study must now be completed to assess the performances of the concept in terms of systematic bias, taking into account all relevant geophysical interferants, and then validating or refining the interferometer design. Therefore, operability of the instrument also needs to be addressed,

\footnotetext{
$\overline{2}$ http://abct.lmd.polytechnique.fr/.
}

by taking into account instrumental and platform-induced issues, as well as in-orbit calibration capabilities. An experimental proof of concept is planned in the framework of the SCARBO project.

In conclusion, it is interesting to note that the NanoCarb concept is very flexible at the design level, allowing to target various spectral signatures and therefore several different species in the atmosphere. In each case, the instrument is spectrally and interferometrically optimized for the latter, assuming a good knowledge of what is to be measured. This feature is well suited for trace gasses in the Earth's atmosphere. Thus, NanoCarb concept is more a $\mathrm{CO}_{2}$ and $\mathrm{CH}_{4}$-dedicated sensor than a classic spectrometer, which is unusual in earth observation, but a key feature of miniaturization.

Acknowledgements This project has received funding from the European Union's H2020 research and innovation program under grant agreement No 769032. The authors would like also to specially thank the FOCUS French label of excellence LabEx FOCUS (ANR-11LABX-0013) for their funding on parts of this work, as well as for their involvements in these challenging developments.

Open Access This article is distributed under the terms of the Creative Commons Attribution 4.0 International License (http://creativeco mmons.org/licenses/by/4.0/), which permits unrestricted use, distribution, and reproduction in any medium, provided you give appropriate credit to the original author(s) and the source, provide a link to the Creative Commons license, and indicate if changes were made.

\section{References}

1. Ciais, P., et al.: Current systematic carbon-cycle observations and the need for implementing a policy-relevant carbon observing system. Biogeosciences 11, 3547-3602 (2014)

2. Ehret, G., et al.: MERLIN: a French-German space lidar mission dedicated to atmospheric methane. Remote Sens. 9, 1052 (2017)

3. Singh, U.N., et al.: Feasibility study of a space-based high pulse energy $2 \mu \mathrm{m}$ CO2 IPDA lidar. Appl. Opt. 56, 6531-6546 (2017)

4. Crevoisier, C., et al.: Tropospheric methane in the tropics-first year from IASI hyperspectral infrared observations. Atmos. Chem. Phys. 9, 6337-6350 (2009)

5. Griffith, D.W., et al.: Preliminary validation of column-averaged volume mixing ratios of carbon dioxide and methane retrieved from GOSAT short-wavelength infrared spectra. Atmos. Meas. Tech. 4, 1061-1076 (2011)

6. Pasternak, F., Bernard, P., Georges, L., Pascal, V.: The microcarb instrument. Proc. SPIE 10562, 105621P (2016)

7. Sierk, B., Löscher, A., Caron, J., Bézy, J.-L., Meijer, Y.: CarbonSat instrument pre-developments: towards monitoring carbon dioxide and methane concentration from space. Proc. SPIE 10562, 105622C (2016)

8. SCARBO project website, http://scarbo-h2020.eu/

9. Van Amerongen, A., Rietjens, J., Smit, M., Van Loon, D., Van Brug, H., Van Der Meulen, W., Esposito, M., Hasekamp, O.: Spex the Dutch roadmap towards aerosol measurement from space. Proc. SPIE 10562, 1056210 (2016)

10. Gousset, S., Le Coarer, E., Guérineau, N., Croizé, L., Laveille, T., Ferrec, Y.: NANOCARB-21: a miniature Fourier-Transform spectro-imaging concept for a daily monitoring of greenhouse gas 
concentration on the Earth surface. Proc. SPIE 10562, 105624U (2016)

11. Rodgers, C.D.: Inverse methods for atmospheric sounding: theory and practice, vol. 2. World scientific, Singapore (2000)

12. Ferrec, Y., Bonnery, G., Brooker, L., Croizé, L., Gousset, S., Le Coarer, E.: NanoCarb part 1: compact snapshot imaging interferometer for $\mathrm{CO}_{2}$ monitoring from space. In ICSO 2018. Int. Soc. Opt. Photonics 11180, 1118021 (2018)

13. Kyle, T.G.: Temperature soundings with partially scanned interferograms. Appl. Opt. 16(2), 326-333 (1977)

14. Fortunato, G.: Application of interferential correlation of spectrum to the detection of atmospheric pollutants. J. Opt. 9(5), 281-290 (1978)

15. Pierangelo, C., Hébert, P., Camy-Peyret, C., Clerbaux, C., Coheur, P., Phulpin, T., Lavanant, L., Tremas, T., Henry, P., and Rosak, A.: SIFTI, a Static Infrared Fourier transform Interferometer dedicated to ozone and $\mathrm{CO}$ pollution monitoring, Proc. of 16th International TOVS Study Conferences (ITSC), 375-385 (2008)

16. Grieco, G., Masiello, G., Serio, C.: Interferometric vs spectral IASI radiances: effective data-reduction approaches for the satellite sounding of atmospheric thermodynamical parameters. Int. J. Remote Sens. 2(10), 2323-2346 (2010)

17. Tatulli, E., Millour, F., Chelli, A., Duvert, G., Acke, B., Utrera, O.H., Petrov, R.G.: Interferometric data reduction with AMBER/ VLTI. Principle, estimators, and illustration. Astron. Astrophys. 464(1), 29-42 (2007)

18. Ehrhardt, H., Gousset, S., Boussey, J., Panabière, M., Le Coarer, E., Croizé, L., Ferrec, Y., Brooker, L., The Scarbo Consortium: Characterization by OCT of a new kind of micro-interferometric components for the Nanocarb miniature imaging spectrometer In ICSO 2018. Int. Soc. Opt. Photonics 11180, 1118066 (2018)

19. Rommeluère, S., Haïdar, R., Guérineau, N., Deschamps, J., De Borniol, E., Million, A., Destefanis, G.: Single-scan extraction of two-dimensional parameters of infrared focal plane arrays utilizing a Fourier-Transform spectrometer. Appl. Opt. 46(9), 1379-1384 (2007)

20. Sissenwine, N., Dubin, M., Wexler, H.: The US standard atmosphere, 1962. J. Geophys. Res. 67(9), 3627-3630 (1962)

21. Clough, S.A., Shephard, M.W., Mlawer, E.J., Delamere, J.S., Iacono, M.J., Cady-Pereira, K., Boukabara, S., Brown, P.D.:
Atmospheric radiative transfer modeling: a summary of the AER codes, short communication. J. Quant. Spectrosc. Radiat. Transf. 91, 233-244 (2005)

22. Rothman, et al.: The HITRAN 2012 molecular spectroscopic database. J. Quant. Spectrosc. Radiat. Transf. 130, 4-50 (2013)

23. Buscher, D.F.: Practical optical interferometry, vol. 11, pp. 140150. Cambridge University Press, Cambridge (2015)

24. Delannoy, A., Fièque, B., Chorier, P., Riuné, C.: NGP: a new large format infrared detector for observation, hyperspectral and spectroscopic space missions in VISIR, SWIR and MWIR wavebands. In sensors, systems, and next-generation satellites XIX. Int. Soc. Opt. Photonics 9639, 96390R (2015)

25. Pascal, V., Buil, C., Loesel, J., Tauziede, L., Jouglet, D., Buisson, F.: An improved microcarb dispersive instrumental concept for the measurement of greenhouse gases concentration in the atmosphere. In International Conference on Space Optics4ICSO 2014. Int. Soc. Opt. Photonics 10563, 105633K (2017)

26. Boulade, O., Moreau, V., Mulet, P., Gravrand, O., Cervera, C., Zanatta, J.P., Roumegoux, J.: Development activities on NIR large format MCT detectors for astrophysics and space science at CEA and SOFRADIR. In High Energy, Optical, and Infrared Detectors for Astronomy VII. Int. Soc. Opt. Photonics 9915, 99150C (2016)

27. Ferrec, Y., Coudrain, C., Primot, J., Ayari-Matallah, N., Chavel, P.H., Goudail, F., Fontanella, J.C.L.: Noise sources in imaging static Fourier Transform spectrometers. Opt. Eng. 51(11), 111716 (2012)

28. McKeever, J., Durak, B.O.A., Gains, D., Jervis, D., Varon, D.J., Germain, S., Sloan, J.J.: GHGSat-D: Greenhouse gas plume imaging and quantification from space using a Fabry-Perot imaging spectrometer. AGU Fall Meeting Abstracts 33 (2017)

29. Buchwitz, M., Reuter, M., Bovensmann, H., Pillai, D., Heymann, J., Schneising, O., Rozanov, V., Krings, T., Burrows, J.P., Boesch, H., et al.: Carbon monitoring satellite (CarbonSat): assessment of atmospheric $\mathrm{CO} 2$ and $\mathrm{CH} 4$ retrieval errors by error parameterization. Atmos. Meas. Tech. 6, 3477-3500 (2013)

Publisher's Note Springer Nature remains neutral with regard to jurisdictional claims in published maps and institutional affiliations. 